\title{
On the theory of cubic residues and nonresidues
}

\author{
by
}

\author{
ZHI-Hong Sun (Huaiyin)
}

1. Introduction. Let $\mathbb{Z}$ be the set of integers, $\omega=(-1+\sqrt{-3}) / 2$ and $\mathbb{Z}[\omega]=\{a+b \omega \mid a, b \in \mathbb{Z}\}$. For $\pi=a+b \omega \in \mathbb{Z}[\omega]$ the norm of $\pi$ is given by $N \pi=\pi \bar{\pi}=a^{2}-a b+b^{2}$. When $\pi \equiv 2(\bmod 3)$ we say that $\pi$ is primary.

If $\pi \in \mathbb{Z}[\omega], N \pi>1$ and $\pi \equiv \pm 2(\bmod 3)$ we may write $\pi= \pm \pi_{1} \ldots \pi_{r}$, where $\pi_{1}, \ldots, \pi_{r}$ are primary primes. For $\alpha \in \mathbb{Z}[\omega]$ the cubic Jacobi symbol $\left(\frac{\alpha}{\pi}\right)_{3}$ is defined by

$$
\left(\frac{\alpha}{\pi}\right)_{3}=\left(\frac{\alpha}{\pi_{1}}\right)_{3} \ldots\left(\frac{\alpha}{\pi_{r}}\right)_{3},
$$

where $\left(\frac{\alpha}{\pi_{t}}\right)_{3}$ is the cubic residue character of $\alpha$ modulo $\pi_{t}$ which is given by

$$
\left(\frac{\alpha}{\pi_{t}}\right)_{3}= \begin{cases}0 & \text { if } \pi_{t} \mid \alpha \\ \omega^{i} & \text { if } \alpha^{\left(N \pi_{t}-1\right) / 3} \equiv \omega^{i}\left(\bmod \pi_{t}\right)\end{cases}
$$

According to [IR, pp. 135, 313] the cubic Jacobi symbol has the following properties:

(1.1) If $a, b \in \mathbb{Z}$ and $a+b \omega \equiv 2(\bmod 3)$ then $\left(\frac{\omega}{a+b \omega}\right)_{3}=\omega^{(a+b+1) / 3}$.

(1.2) If $a, b \in \mathbb{Z}$ and $a+b \omega \equiv 2(\bmod 3)$ then $\left(\frac{1-\omega}{a+b \omega}\right)_{3}=\omega^{2(a+1) / 3}$.

(1.3) If $\pi, \lambda \in \mathbb{Z}[\omega]$ and $\pi, \lambda \equiv \pm 2(\bmod 3)$ then $\left(\frac{\lambda}{\pi}\right)_{3}=\left(\frac{\pi}{\lambda}\right)_{3}$.

The assertion (1.3) is now called the general cubic reciprocity law; it was first proved by G. Eisenstein.

Let $p$ be a prime of the form $3 n+1$. It is well known that there are unique integers $L$ and $|M|$ such that $4 p=L^{2}+27 M^{2}$ with $L \equiv 1(\bmod 3)$. It follows that $\left(\frac{L}{3 M}\right)^{2} \equiv-3(\bmod p)$ and therefore $m^{(p-1) / 3} \equiv 1,\left(-1-\frac{L}{3 M}\right) / 2$ or $\left(-1+\frac{L}{3 M}\right) / 2(\bmod p)$ for any integer $m \not \equiv 0(\bmod p)$.

In 1827 Jacobi $[\mathrm{J}]$ established the following rational cubic reciprocity law.

1991 Mathematics Subject Classification: 11A15, 11E25. 
TheOREM 1.1 (Jacobi). Let $q$ be a prime of the form $3 n+1, q \neq p$ and $4 q=L^{\prime 2}+27 M^{\prime 2}$. Then $q$ is a cubic residue modulo $p$ if and only if $\left(L M^{\prime}-L^{\prime} M\right) /\left(L M^{\prime}+L^{\prime} M\right)$ is a cubic residue modulo $q$.

In 1958, using the period equation of degree 3, E. Lehmer [L1] gave the following criterion for cubic residuacity.

THEOREM 1.2 (E. Lehmer). If $q$ is an odd prime different from $p$ then $q$ is a cubic residue of $p$ if and only if either $L M \equiv 0(\bmod q)$ or $L \equiv \mu M$ $(\bmod q)$, where $\mu$ satisfies the congruence

$$
\mu^{2} \equiv \frac{3 u+1}{3 u-3}\left(\frac{9}{2 u+1}\right)^{2}(\bmod q)
$$

with $u \not \equiv 0,1,-\frac{1}{2},-\frac{1}{3}(\bmod q)$ and $\left(\frac{(3 u+1)(3 u-3)}{q}\right)=1$. Here $(\dot{\bar{q}})$ is the Legendre symbol.

In 1975 K. S. Williams [W1] showed how to choose the sign of $M$ so that $m^{(p-1) / 3} \equiv\left(-1-\frac{L}{3 M}\right) / 2(\bmod p)$ when $m$ is a cubic nonresidue modulo $p$.

Let $\varepsilon_{d}$ be the fundamental unit in the quadratic field $\mathbb{Q}(\sqrt{d})$. In 1970's E. Lehmer [L3], [L4] began to study criteria for $\varepsilon_{d}$ to be a cubic residue modulo $p$, where $p$ is a prime of the form $3 n+1$ satisfying $\left(\frac{d}{p}\right)=1$.

Since the work of Euler, Gauss, Jacobi and Eisenstein (see [IR, p. 133]) it is known that cubic congruences are connected with binary quadratic forms. In 1992 B. K. Spearman and K. S. Williams [SW] showed that $m$ is a cubic residue modulo $p$ if and only if $p$ can be represented by one of the third (composition) powers of primitive integral binary quadratic forms of discriminant $-27 m^{2}$, where $p$ is a prime greater than 3 for which $m \neq 0$ $(\bmod p)$.

Let $m$ be a positive integer, and $\mathbb{Z}_{m}$ the set of those rational numbers whose denominator is prime to $m$. Inspired by the above work of Jacobi, Lehmer and Williams we introduce the subsets $C_{0}(m), C_{1}(m)$ and $C_{2}(m)$ of $\mathbb{Z}_{m}$ for $m \not \equiv 0(\bmod 3)$, where

$$
C_{i}(m)=\left\{k \mid\left(\frac{k+1+2 \omega}{m}\right)_{3}=\omega^{i}, k \in \mathbb{Z}_{m}\right\} \quad \text { for } i=0,1,2 .
$$

In Sections 2 and 3 we concentrate on the structure and properties of $C_{0}(m), C_{1}(m)$ and $C_{2}(m)$. Here are some typical results:

(1.4) Let $p$ be a prime of the form $3 n+1$ and hence $4 p=L^{2}+27 M^{2}$ for some $L, M \in \mathbb{Z}$ and $L \equiv 1(\bmod 3)$. If $q$ is a prime such that $M \not \equiv 0$ $(\bmod q)$ and $i \in\{0,1,2\}$ then $q^{(p-1) / 3} \equiv\left(\left(-1-\frac{L}{3 M}\right) / 2\right)^{i}(\bmod p)$ if and only if $L /(3 M) \in C_{i}(q)$. 
(1.5) Let $p$ be a prime for which $p \equiv 1(\bmod 3), t^{2} \equiv-3(\bmod p)(t \in \mathbb{Z})$, $k \in \mathbb{Z}_{p}, k^{2}+3 \not \equiv 0(\bmod p)$ and $i \in\{0,1,2\}$. Then $k \in C_{i}(p)$ if and only if

$$
\left(\frac{k-t}{k+t}\right)^{(p-1) / 3} \equiv\left(\frac{-1-t}{2}\right)^{i}(\bmod p) .
$$

(1.6) Let $p$ be a prime greater than $3, k \in \mathbb{Z}_{p}$ and $k^{2}+3 \not \equiv 0(\bmod p)$. Then $k \in C_{0}(p)$ if and only if

$$
k \equiv \frac{x^{3}-9 x}{3 x^{2}-3}(\bmod p) \quad \text { for some integer } x .
$$

If $q$ is also a prime of the form $3 n+1$ and $4 q=L^{\prime 2}+27 M^{\prime 2}\left(L^{\prime}, M^{\prime} \in \mathbb{Z}\right)$ with $L^{\prime} \equiv 1(\bmod 3)$, in view of $(1.4)$ and $(1.5)$ we see that

$$
q^{(p-1) / 3} \equiv\left(\frac{-1-L /(3 M)}{2}\right)^{i}(\bmod p)
$$

if and only if

$$
\left(\frac{L M^{\prime}-L^{\prime} M}{L M^{\prime}+L^{\prime} M}\right)^{(q-1) / 3} \equiv\left(\frac{-1-L^{\prime} /\left(3 M^{\prime}\right)}{2}\right)^{i}(\bmod q) .
$$

This generalizes Jacobi's result.

Combining (1.4) with (1.6) gives a simple criterion for cubic residuacity which improves Lehmer's result.

Section 4 is devoted to cubic congruences. Here are two main results:

(1.7) If $p>3$ is a prime, $a, b \in \mathbb{Z}_{p}, p \nmid a b$ and $s^{2} \equiv-3\left(b^{2}-4 a\right)(\bmod p)$ for some $s \in \mathbb{Z}$, then the congruence $x^{3}-3 a x-a b \equiv 0(\bmod p)$ is solvable if and only if $s / b \in C_{0}(p)$.

(1.8) Suppose that $p$ is a prime greater than 3 and that $N$ is the number of values of $x^{3}+A x^{2}+B x+C$ modulo $p$, where $A, B, C \in \mathbb{Z}$ and $x$ runs over all integers. If $A^{2} \not \equiv 3 B(\bmod p)$ then $N=p-\left(p-\left(\frac{-3}{p}\right)\right) / 3$. If $A^{2} \equiv 3 B$ $(\bmod p)$ then $N=(p+2) / 3$ or $p$ according as $p \equiv 1(\bmod 3)$ or $p \equiv 2$ $(\bmod 3)$.

In Section 5 the criteria for $s(d) \in C_{i}(p)(i=0,1,2)$ are given in terms of binary quadratic forms, where $p>3$ is a prime, $d \in \mathbb{Z}, p \nmid(d+3)$ and $(s(d))^{2} \equiv d(\bmod p)$. In particular, sufficient and necessary conditions for $s(d) \in C_{0}(p)$ are described in the cases $d=-1,-2,-5,-6,-7$ and -15 . As a consequence we obtain criteria for $\varepsilon_{6}, \varepsilon_{15}, \varepsilon_{21}$ to be cubic residues modulo $p$.

In Section 6 we mainly determine $u_{\left(p-\left(\frac{-3}{p}\right)\right) / 3}(a, b)$ modulo $p$, where $p>3$ is a prime and $\left\{u_{n}(a, b)\right\}$ is the Lucas sequence given by $u_{0}(a, b)=0$, $u_{1}(a, b)=1$ and $u_{n+1}(a, b)=b u_{n}(a, b)-a u_{n-1}(a, b)(n \geq 1)$. In particular, we obtain $F_{\left(p-\left(\frac{-3}{p}\right)\right) / 3}(\bmod p)$ and $P_{\left(p-\left(\frac{-3}{p}\right)\right) / 3}(\bmod p)$, where $\left\{F_{n}\right\}$ and $\left\{P_{n}\right\}$ denote the Fibonacci sequence and Pell sequence respectively. 
To illustrate the connections in the above work I state the following result:

(1.9) Let $p$ be a prime for which $\left(\frac{-3}{p}\right)=\left(\frac{5}{p}\right)=1$, and $q$ a prime of the form $3 n+1$ satisfying $L^{2}+135 M^{2} \equiv 0(\bmod p)$, where $L$ and $M$ are determined by $4 q=L^{2}+27 M^{2}(L, M \in \mathbb{Z})$. Then the following statements are equivalent:

(a) $p$ is a cubic residue modulo $q$.

(b) $s(-15) \in C_{0}(p)$.

(c) $\varepsilon_{5}(=(1+\sqrt{5}) / 2)$ is a cubic residue modulo $p$.

(d) The congruence $x^{3}+3 x+1 \equiv 0(\bmod p)$ is solvable.

(e) $p \mid F_{(p-1) / 3}$.

(f) $p=x^{2}+135 y^{2}$ for some integers $x$ and $y$.

For later convenience we list the following notations:

$\omega=(-1+\sqrt{-3}) / 2, \mathbb{Z}$ - the set of integers, $\mathbb{Z}^{+}$- the set of natural numbers, $\mathbb{Z}[\omega]$ - the set $\{a+b \omega \mid a, b \in \mathbb{Z}\}, N \pi$ - the norm of $\pi, \mathbb{Q}$ - the set of rational numbers, $\mathbb{Z}_{m}$ - the set of those rational numbers whose denominator is prime to $m,[x]$ - the greatest integer not exceeding $x,[x]_{p}$ - the set $\left\{k \mid k \equiv x(\bmod p), k \in \mathbb{Z}_{p}\right\},(a, b)$-the greatest common divisor of $a$ and $b,[a, b]$ - the least common multiple of $a$ and $b, m \mid n-m$ divides $n$, $m \nmid n-m$ does not divide $n,\left(\frac{a}{p}\right)$ - the Legendre symbol, $\left(\frac{\alpha}{\pi}\right)_{3}$ - the cubic Jacobi symbol.

2. Basic properties of $C_{i}(m)$. Let $m \in \mathbb{Z}^{+}$and $m \neq \equiv 0(\bmod 3)$. For $a, b \in \mathbb{Z}_{m}$ it is clear that there are unique integers $a_{0}, b_{0} \in\{0,1, \ldots, m-1\}$ satisfying $a \equiv a_{0}(\bmod m)$ and $b \equiv b_{0}(\bmod m)$. From this we may define

$$
(a, m)=\left(a_{0}, m\right) \text { and }\left(\frac{a+b \omega}{m}\right)_{3}=\left(\frac{a_{0}+b_{0} \omega}{m}\right)_{3} \text { for } m>1 .
$$

When $m=1$ define

$$
(a, m)=1 \text { and }\left(\frac{a+b \omega}{m}\right)_{3}=1 .
$$

One can easily verify the following facts:

(2.1) If $a, b, c, d \in \mathbb{Z}_{m}$ then

$$
\left(\frac{a+b \omega}{m}\right)_{3}\left(\frac{c+d \omega}{m}\right)_{3}=\left(\frac{(a+b \omega)(c+d \omega)}{m}\right)_{3} .
$$

(2.2) If $n \in \mathbb{Z}_{m}$ and $(m, n)=1$ then $\left(\frac{n}{m}\right)_{3}=1$.

(2.3) If $a, b \in \mathbb{Z}_{m_{1} m_{2}}$ then

$$
\left(\frac{a+b \omega}{m_{1} m_{2}}\right)_{3}=\left(\frac{a+b \omega}{m_{1}}\right)_{3}\left(\frac{a+b \omega}{m_{2}}\right)_{3} .
$$


Definition 2.1. Suppose $m \in \mathbb{Z}^{+}$and $m \not \equiv 0(\bmod 3)$. For $i=0,1,2$ define

$$
C_{i}(m)=\left\{k \mid\left(\frac{k+1+2 \omega}{m}\right)_{3}=\omega^{i}, k \in \mathbb{Z}_{m}\right\} .
$$

From the above definition it is easy to prove the following results:

(2.4) $C_{0}(m) \cup C_{1}(m) \cup C_{2}(m)=\left\{k \mid\left(k^{2}+3, m\right)=1, k \in \mathbb{Z}_{m}\right\}$.

(2.5) $k \in C_{0}(m)$ if and only if $-k \in C_{0}(m)$.

(2.6) $k \in C_{1}(m)$ if and only if $-k \in C_{2}(m)$.

ExAmple 2.1. Set $C_{i}^{*}(m)=C_{i}(m) \cap\{k \mid-m / 2<k \leq m / 2, k \in \mathbb{Z}\}$ for $i=0,1,2$. Then

$$
\begin{aligned}
C_{0}^{*}(5) & =\{0\}, & C_{1}^{*}(5) & =\{1,2\} ; \\
C_{0}^{*}(7) & =\{0\}, & C_{1}^{*}(7) & =\{-1,3\} ; \\
C_{0}^{*}(11) & =\{0,5,-5\}, & C_{1}^{*}(11) & =\{-1,-2,3,-4\} ; \\
C_{0}^{*}(13) & =\{0,4,-4\}, & C_{1}^{*}(13) & =\{1,-2,-3,-5\} ; \\
C_{0}^{*}(17) & =\{0,1,-1,3,-3\}, & C_{1}^{*}(17) & =\{2,4,-5,-6,7,-8\} ; \\
C_{0}^{*}(19) & =\{0,1,-1,3,-3\}, & C_{1}^{*}(19) & =\{-2,5,-6,7,-8,-9\} .
\end{aligned}
$$

Proposition 2.1. Suppose $m \in \mathbb{Z}^{+}$and $m \not \equiv 0(\bmod 3)$. Then $0 \in$ $C_{0}(m)$.

\section{Pro of. Since}

$$
\left(\frac{1+2 \omega}{m}\right)_{3}=\left(\frac{1+2 \omega}{m}\right)_{3}^{4}=\left(\frac{(1+2 \omega)^{4}}{m}\right)_{3}=\left(\frac{9}{m}\right)_{3}=1
$$

we see that $0 \in C_{0}(m)$.

Lemma 2.1. Suppose that $m \in \mathbb{Z}^{+}, m \neq \equiv 0(\bmod 3), k_{1}, k_{2} \in \mathbb{Z}_{m}$, $\left(\left(k_{1}^{2}+3\right)\left(k_{2}^{2}+3\right), m\right)=1$, and $m^{\prime}$ is the greatest divisor of $m$ for which $\left(m^{\prime}, k_{1}+k_{2}\right)=1$. Then

$$
\left(\frac{k_{1}+1+2 \omega}{m}\right)_{3}\left(\frac{k_{2}+1+2 \omega}{m}\right)_{3}=\left(\frac{\frac{k_{1} k_{2}-3}{k_{1}+k_{2}}+1+2 \omega}{m^{\prime}}\right)_{3} .
$$

Proof. Since $\left(k_{1}+1+2 \omega\right)\left(k_{2}+1+2 \omega\right)=k_{1} k_{2}-3+\left(k_{1}+k_{2}\right)(1+2 \omega)$ it is seen that

$$
\begin{aligned}
& \left(\frac{k_{1}+1+2 \omega}{m}\right)_{3}\left(\frac{k_{2}+1+2 \omega}{m}\right)_{3} \\
& \quad=\left(\frac{k_{1} k_{2}-3+\left(k_{1}+k_{2}\right)(1+2 \omega)}{m}\right)_{3} \\
& \quad=\left(\frac{\frac{k_{1} k_{2}-3}{k_{1}+k_{2}}+1+2 \omega}{m^{\prime}}\right)_{3}\left(\frac{k_{1} k_{2}-3+\left(k_{1}+k_{2}\right)(1+2 \omega)}{m / m^{\prime}}\right)_{3} .
\end{aligned}
$$


When $m=m^{\prime}$, we have

$$
\left(\frac{k_{1} k_{2}-3+\left(k_{1}+k_{2}\right)(1+2 \omega)}{m / m^{\prime}}\right)_{3}=1 .
$$

Now assume that $m>m^{\prime}$ and that $p$ is a prime divisor of $m / m^{\prime}$. It is clear that $k_{1}+k_{2} \equiv 0(\bmod p)$ and therefore that

$$
\left(\frac{k_{1} k_{2}-3+\left(k_{1}+k_{2}\right)(1+2 \omega)}{p}\right)_{3}=\left(\frac{k_{1} k_{2}-3}{p}\right)_{3}=\left(\frac{-k_{1}^{2}-3}{p}\right)_{3}=1 \text {. }
$$

Thus,

$$
\begin{aligned}
\left(\frac{k_{1} k_{2}-3+\left(k_{1}+k_{2}\right)(1+2 \omega)}{m / m^{\prime}}\right)_{3} & \\
= & \prod_{p \mid m / m^{\prime}}\left(\frac{k_{1} k_{2}-3+\left(k_{1}+k_{2}\right)(1+2 \omega)}{p}\right)_{3}=1 .
\end{aligned}
$$

This completes the proof.

Proposition 2.2. Let $m$ be a positive integer not divisible by 3 , and $i \in\{0,1,2\}$.

(i) If $k, k^{\prime} \in \mathbb{Z}_{m}$ and $k k^{\prime} \equiv-3(\bmod m)$ then $k \in C_{i}(m)$ if and only if $k^{\prime} \in C_{i}(m)$.

(ii) If $k_{1}, k_{2} \in C_{i}(m)$ and $\left(k_{1}+k_{2}, m\right)=1$ then $\left(3-k_{1} k_{2}\right) /\left(k_{1}+k_{2}\right) \in$ $C_{i}(m)$.

Pr o of. Since $(k, m)=1$, by Proposition 2.1 we have

$$
\begin{aligned}
\left(\frac{k^{\prime}+1+2 \omega}{m}\right)_{3} & =\left(\frac{k}{m}\right)_{3}\left(\frac{k^{\prime}+1+2 \omega}{m}\right)_{3}=\left(\frac{-3+k+2 k \omega}{m}\right)_{3} \\
& =\left(\frac{1+2 \omega}{m}\right)_{3}\left(\frac{k+1+2 \omega}{m}\right)_{3}=\left(\frac{k+1+2 \omega}{m}\right)_{3} .
\end{aligned}
$$

So (i) follows.

To prove (ii), we note that

$$
\begin{aligned}
\left(\frac{\frac{3-k_{1} k_{2}}{k_{1}+k_{2}}+1+2 \omega}{m}\right)_{3} & =\left(\frac{\frac{k_{1} k_{2}-3}{k_{1}+k_{2}}-1-2 \omega}{m}\right)_{3} \\
& \left.=\frac{\left.\left(\frac{\frac{k_{1} k_{2}-3}{k_{1}+k_{2}}+1+2 \omega}{m}\right)_{3}=\frac{\left(\frac{k_{1} k_{2}-3}{k_{1}+k_{2}}+1+2 \omega\right.}{m}\right)_{3}}{\left(\frac{k_{1}+1+2 \omega}{m}\right)_{3}} \frac{k_{2}+1+2 \omega}{m}\right)_{3} \\
& =(\text { by Lemma 2.1) } \\
& =\overline{\omega^{i} \cdot \overline{\omega^{i}}=\omega^{i} .}
\end{aligned}
$$


Proposition 2.3. Let $m \in \mathbb{Z}^{+}$with $m \neq \equiv 0(\bmod 3)$, and $k \in \mathbb{Z}_{m}$ with $\left(\left(k^{2}-1\right)\left(k^{2}+3\right), m\right)=1$. Then $\left(k^{3}-9 k\right) /\left(3 k^{2}-3\right) \in C_{0}(m)$.

Proof. Clearly,

$(k+1+2 \omega)^{3}=(k+1+2 \omega)\left(k^{2}-3+2 k(1+2 \omega)\right)=k^{3}-9 k+\left(3 k^{2}-3\right)(1+2 \omega)$.

Thus,

$$
\begin{aligned}
\left(\frac{\frac{k^{3}-9 k}{3 k^{2}-3}+1+2 \omega}{m}\right)_{3} & =\left(\frac{k^{3}-9 k+\left(3 k^{2}-3\right)(1+2 \omega)}{m}\right)_{3} \\
& =\left(\frac{(k+1+2 \omega)^{3}}{m}\right)_{3}=1 .
\end{aligned}
$$

The proof is now complete.

Proposition 2.4. Let $m_{1}, m_{2} \in \mathbb{Z}^{+}$be such that $m_{1} m_{2} \not \equiv 0(\bmod 3)$, $k \in \mathbb{Z}$ and $i \in\{0,1,2\}$. If $m_{1} \equiv m_{2}\left(\bmod \left[9, k^{2}+3\right]\right)$ then $k \in C_{i}\left(m_{1}\right)$ if and only if $k \in C_{i}\left(m_{2}\right)$.

Proof. Write $k+1+2 \omega=(-1)^{j} \omega^{s}(1-\omega)^{t} \pi_{1} \ldots \pi_{r}$, where $\pi_{1}, \ldots, \pi_{r}$ are primary primes in $\mathbb{Z}[\omega]$. Since $(k+1+2 \omega)\left(k+1+2 \omega^{2}\right)=k^{2}+3$ it is seen that $k^{2}+3 \equiv 0\left(\bmod \pi_{i}\right)(i=1, \ldots, r)$. Using Proposition 2.1 and (1.1) we find

$$
\left(\frac{\omega(1-\omega)}{m_{1}}\right)_{3}=\left(\frac{\omega(1-\omega)}{m_{2}}\right)_{3}=1 \text { and }\left(\frac{\omega}{m_{1}}\right)_{3}=\left(\frac{\omega}{m_{2}}\right)_{3} .
$$

Hence,

$$
\begin{aligned}
\left(\frac{k+1+2 \omega}{m_{1}}\right)_{3} & =\left(\frac{(-1)^{j} \omega^{s-t}}{m_{1}}\right)_{3}\left(\frac{\omega(1-\omega)}{m_{1}}\right)_{3}^{t} \prod_{i=1}^{r}\left(\frac{\pi_{i}}{m_{1}}\right)_{3} \\
& =\left(\frac{\omega}{m_{1}}\right)_{3}^{s-t} \prod_{i=1}^{r}\left(\frac{m_{1}}{\pi_{i}}\right)_{3}=\left(\frac{\omega}{m_{2}}\right)_{3}^{s-t} \prod_{i=1}^{r}\left(\frac{m_{2}}{\pi_{i}}\right)_{3} \\
& =\left(\frac{k+1+2 \omega}{m_{2}}\right)_{3} .
\end{aligned}
$$

This proves the result.

Now we point out the connections between $C_{i}(m)(i \in\{0,1,2\})$ and cubic residues.

Theorem 2.1. Let $p \equiv 1(\bmod 3)$ be a prime $4 p=L^{2}+27 M^{2}(L, M \in$ $\mathbb{Z})$ with $L \equiv 1(\bmod 3)$, and $m=2^{\alpha} 3^{\beta} m^{\prime} m^{\prime \prime} \in \mathbb{Z}^{+}$with $m^{\prime}=\max \{d|d| m$, $(d, 6 M)=1\}$ and $\left(6, m^{\prime \prime}\right)=1$. Then, for $i=0,1,2$,

$$
m^{(p-1) / 3} \equiv\left(\frac{-1-L /(3 M)}{2}\right)^{i}(\bmod p)
$$


if and only if $L /(3 M) \in C_{i^{\prime}}\left(m^{\prime}\right)$, where $i^{\prime} \in\{0,1,2\}$ is determined by

$$
i^{\prime} \equiv\left\{\begin{aligned}
& i+\beta M(\bmod 3) \text { if } 3 \mid \alpha \text { or } 2 \mid M \\
& i+\beta M+(-1)^{r+s}(\bmod 3) \\
& \text { if } \alpha \equiv(-1)^{r}(\bmod 3) \text { and } L \equiv(-1)^{s-1} M(\bmod 4) .
\end{aligned}\right.
$$

Proof. Set $\pi=(L+3 M) / 2+3 M \omega$. Then $\pi \in \mathbb{Z}[\omega]$. Clearly $\pi \equiv 2$ $(\bmod 3)$ and $N \pi=p$. Thus,

$$
\begin{aligned}
m^{(p-1) / 3} & \equiv\left(\frac{-1-L /(3 M)}{2}\right)^{i}(\bmod p) \\
& \Leftrightarrow m^{(p-1) / 3} \equiv\left(\frac{-1-L /(3 M)}{2}\right)^{i} \equiv \omega^{i}(\bmod \pi) \\
& \Leftrightarrow\left(\frac{2^{\alpha} m^{\prime} m^{\prime \prime}}{\pi}\right)_{3}\left(\frac{\omega(1-\omega)}{\pi}\right)_{3}^{2 \beta}=\left(\frac{m}{\pi}\right)_{3}=\omega^{i} \\
& \Leftrightarrow\left(\frac{\pi}{2^{\alpha} m^{\prime} m^{\prime \prime}}\right)_{3}=\left(\frac{2^{\alpha} m^{\prime} m^{\prime \prime}}{\pi}\right)_{3}=\omega^{i-2 \beta M}=\omega^{i+\beta M}
\end{aligned}
$$

(by (1.1), (1.2) and (1.3)).

Now let us calculate $\left(\frac{\pi}{m^{\prime \prime}}\right)_{3}$. Obviously $\left(\frac{\pi}{m^{\prime \prime}}\right)_{3}=1$ for $m^{\prime \prime}=1$. Assume that $m^{\prime \prime}>1$ and that $q$ is a prime divisor of $m^{\prime \prime}$. It is clear that $q \mid M$ and so that $q \nmid L$. Thus,

$$
\left(\frac{\pi}{m^{\prime \prime}}\right)_{3}=\prod_{q \mid m^{\prime \prime}}\left(\frac{(L+3 M) / 2+3 M \omega}{q}\right)_{3}=\prod_{q \mid m^{\prime \prime}}\left(\frac{L / 2}{q}\right)_{3}=1 .
$$

On the other hand,

$$
\left(\frac{(L+3 M) / 2+3 M \omega}{2}\right)_{3}=\left\{\begin{array}{l}
\left(\frac{(L+3 M) / 2}{2}\right)_{3} \text { if } 2 \mid M, \\
\left(\frac{3 M \omega}{2}\right)_{3}=\left(\frac{\omega}{2}\right)_{3}=\omega \\
\text { if } 2 \nmid M \text { and } L \equiv M(\bmod 4), \\
\left(\frac{1+\omega}{2}\right)_{3}=\left(\frac{\omega}{2}\right)_{3}^{2}=\omega^{-1} \\
\text { if } 2 \nmid M \text { and } L \equiv-M(\bmod 4) .
\end{array}\right.
$$

So

$$
\left(\frac{\pi}{2^{\alpha}}\right)_{3}=\left(\frac{\pi}{2}\right)_{3}^{\alpha}=\left\{\begin{array}{l}
1 \quad \text { if } 3 \mid \alpha \text { or } 2 \mid M \\
\left(\omega^{(-1)^{s-1}}\right)^{\alpha}=\omega^{-(-1)^{s+r}} \\
\quad \text { if } 3 \mid\left(\alpha-(-1)^{r}\right) \text { and } 4 \mid\left(L-(-1)^{s-1} M\right) .
\end{array}\right.
$$


Putting the above together we see that

$m^{(p-1) / 3} \equiv\left(\frac{-1-L /(3 M)}{2}\right)^{i}(\bmod p) \Leftrightarrow\left(\frac{\pi}{m^{\prime}}\right)_{3}=\omega^{i+\beta M}\left(\frac{\pi}{2^{\alpha}}\right)_{3}^{-1}=\omega^{i^{\prime}}$.

This concludes the proof.

COROLlary 2.1. Let $p$ and $q$ be distinct primes greater than $3, p \equiv 1$ $(\bmod 3)$ and $4 p=L^{2}+27 M^{2}(L, M \in \mathbb{Z})$ with $L \equiv 1(\bmod 3)$. If $q \mid M$ then $q^{(p-1) / 3} \equiv 1(\bmod p)$. If $q \nmid M$ and $i \in\{0,1,2\}$ then

$$
q^{(p-1) / 3} \equiv\left(\frac{-1-L /(3 M)}{2}\right)^{i}(\bmod p)
$$

if and only if $L /(3 M) \in C_{i}(q)$.

REMARK 2.1. According to Theorem 2.1 the value of $m^{(p-1) / 3}(\bmod p)$ can be completely determined. The special cases $m=2,3$ were treated by E. Lehmer [L2] and K. S. Williams [W1] respectively. When $m$ is a prime for which $m \neq 2,3, p$, it follows from Corollary 2.1 that $m^{(p-1) / 3}(\bmod p)$ depends only on $L /(3 M)(\bmod m)$. This important fact was first observed by Jacobi $[\mathrm{J}]$, and proved by E. Lehmer [L1] and K. S. Williams [W1].

LEMMA 2.2. Let $p \neq 3$ be a prime and $k \in \mathbb{Z}_{p}$.

(i) If $p \equiv 1(\bmod 3)$ and so $p=\lambda \bar{\lambda}$ with $\lambda \in \mathbb{Z}[\omega]$ and $\lambda \equiv 2(\bmod 3)$ then

$$
\left(\frac{k+1+2 \omega}{p}\right)_{3}=\left(\frac{\left(k^{2}+3\right)(k-1-2 \omega)}{\lambda}\right)_{3} .
$$

(ii) If $p \equiv 2(\bmod 3)$ then

$$
\left(\frac{k+1+2 \omega}{p}\right)_{3} \equiv\left(k^{2}+3\right)^{(p-2) / 3}(k+1+2 \omega)^{(p+1) / 3}(\bmod p) .
$$

Proof. Suppose $p=\lambda \bar{\lambda} \equiv 1(\bmod 3)$ with $\lambda \in \mathbb{Z}[\omega]$ and $\lambda \equiv 2(\bmod 3)$. From the properties of the cubic residue character it is seen that

$$
\begin{aligned}
\left(\frac{k+1+2 \omega}{p}\right)_{3} & =\left(\frac{k+1+2 \omega}{\lambda}\right)_{3}\left(\frac{k+1+2 \omega}{\bar{\lambda}}\right)_{3} \\
& =\left(\frac{k+1+2 \omega}{\lambda}\right)_{3}\left(\frac{k-1-2 \omega}{\lambda}\right)_{3} \\
& =\left(\frac{k+1+2 \omega}{\lambda}\right)_{3}\left(\frac{k-1-2 \omega}{\lambda}\right)_{3}^{2} \\
& =\left(\frac{\left(k^{2}+3\right)(k-1-2 \omega)}{\lambda}\right)_{3} .
\end{aligned}
$$

For (ii), we note that

$(k+1+2 \omega)^{p} \equiv(k+1)^{p}+2^{p} \omega^{p} \equiv k+1+2 \omega^{2}=k-1-2 \omega(\bmod p)$ 
and so

$$
\begin{aligned}
& \left(\frac{k+1+2 \omega}{p}\right)_{3} \\
& \quad \equiv(k+1+2 \omega)^{\left(p^{2}-1\right) / 3}=(k+1+2 \omega)^{\frac{p(p-2)}{3}+\frac{p-2}{3}+\frac{p+1}{3}} \\
& \quad \equiv(k-1-2 \omega)^{(p-2) / 3}(k+1+2 \omega)^{(p-2) / 3}(k+1+2 \omega)^{(p+1) / 3} \\
& \quad=\left(k^{2}+3\right)^{(p-2) / 3}(k+1+2 \omega)^{(p+1) / 3}(\bmod p) .
\end{aligned}
$$

Now we are ready to give

Theorem 2.2. Let $p \neq 3$ be a prime, $i \in\{0,1,2\}$ and $k \in \mathbb{Z}_{p}$ with $k^{2}+3 \not \equiv 0(\bmod p)$.

(i) If $p \equiv 1(\bmod 3)$ and so $t^{2} \equiv-3(\bmod p)$ for some $t \in \mathbb{Z}_{p}$ then $k \in C_{i}(p)$ if and only if

$$
\left(\frac{k-t}{k+t}\right)^{(p-1) / 3} \equiv\left(\frac{-1-t}{2}\right)^{i}(\bmod p) .
$$

(ii) If $p \equiv 2(\bmod 3)$ then $k \in C_{i}(p)$ if and only if

$$
\left(\frac{k-1-2 \omega}{k+1+2 \omega}\right)^{(p+1) / 3} \equiv \omega^{i}(\bmod p) .
$$

Proof. Suppose $p \equiv 1(\bmod 3), 4 p=L^{2}+27 M^{2}(L, M \in \mathbb{Z})$ and $L \equiv 1$ $(\bmod 3)$. Since $(L /(3 M))^{2} \equiv t^{2} \equiv-3(\bmod p)$ we may choose $M$ so that $L \equiv 3 M t(\bmod p)$. Set $\lambda=(L+3 M) / 2+3 M \omega$. Then $\lambda \in \mathbb{Z}[\omega]$ and $\lambda \equiv 2$ $(\bmod 3)$. Clearly $N \lambda=p$ and

$$
\omega \equiv \frac{-1-L /(3 M)}{2}(\bmod \lambda) .
$$

Thus, by Lemma 2.2 we have

$$
\begin{aligned}
\left(\frac{k+1+2 \omega}{p}\right)_{3} & =\left(\frac{\left(k^{2}+3\right)(k-1-2 \omega)}{\lambda}\right)_{3} \\
& \equiv\left(\left(k^{2}+3\right)(k-1-2 \omega)\right)^{(p-1) / 3} \\
& \equiv\left(\left(k^{2}+3\right)\left(k+\frac{L}{3 M}\right)\right)^{(p-1) / 3} \\
& \equiv\left((k+t)^{2}(k-t)\right)^{(p-1) / 3}(\bmod \lambda) .
\end{aligned}
$$

It then follows that

$$
\begin{aligned}
k \in C_{i}(p) & \Leftrightarrow\left(\frac{k+1+2 \omega}{p}\right)_{3}=\omega^{i} \\
& \Leftrightarrow\left((k+t)^{2}(k-t)\right)^{(p-1) / 3} \equiv\left(\frac{-1-t}{2}\right)^{i}(\bmod \lambda)
\end{aligned}
$$




$$
\begin{aligned}
& \Leftrightarrow\left((k+t)^{2}(k-t)\right)^{(p-1) / 3} \equiv\left(\frac{-1-t}{2}\right)^{i}(\bmod p) \\
& \Leftrightarrow\left(\frac{k-t}{k+t}\right)^{(p-1) / 3} \equiv\left(\frac{-1-t}{2}\right)^{i}(\bmod p) .
\end{aligned}
$$

This proves (i).

Now consider (ii). Note that $(k+1+2 \omega)^{p} \equiv k-1-2 \omega(\bmod p)$. Using Lemma 2.2 we see that

$$
\begin{aligned}
\left(\frac{k+1+2 \omega}{p}\right)_{3} & \equiv(k-1-2 \omega)^{(p-2) / 3}(k+1+2 \omega)^{(p-2) / 3}(k+1+2 \omega)^{(p+1) / 3} \\
& =\left(\frac{k-1-2 \omega}{k+1+2 \omega}\right)^{(p-2) / 3}(k+1+2 \omega)^{\frac{2(p-2)}{3}+\frac{p+1}{3}} \\
& =\left(\frac{k-1-2 \omega}{k+1+2 \omega}\right)^{(p+1) / 3} \frac{k+1+2 \omega}{k-1-2 \omega}(k+1+2 \omega)^{p-1} \\
& \equiv\left(\frac{k-1-2 \omega}{k+1+2 \omega}\right)^{(p+1) / 3}(\bmod p) .
\end{aligned}
$$

This completes the proof.

From Theorem 2.2 we have the following rational cubic reciprocity law.

Corollary 2.2. Let $p$ and $q$ be distinct primes, $p \equiv 1(\bmod 3), 4 p=$ $L^{2}+27 M^{2}(L, M \in \mathbb{Z}), L \equiv 1(\bmod 3), q>3$ and $i \in\{0,1,2\}$.

(i) If $q \equiv 1(\bmod 3)$ and hence $4 q=L^{\prime 2}+27 M^{\prime 2}\left(L^{\prime}, M^{\prime} \in \mathbb{Z}\right)$ then

$$
q^{(p-1) / 3} \equiv\left(\frac{-1-L /(3 M)}{2}\right)^{i}(\bmod p)
$$

if and only if

$$
\left(\frac{L M^{\prime}-L^{\prime} M}{L M^{\prime}+L^{\prime} M}\right)^{(q-1) / 3} \equiv\left(\frac{-1-L^{\prime} /\left(3 M^{\prime}\right)}{2}\right)^{i}(\bmod q) .
$$

(ii) If $q \equiv 2(\bmod 3)$ then

$$
q^{(p-1) / 3} \equiv\left(\frac{-1-L /(3 M)}{2}\right)^{i}(\bmod p)
$$

if and only if

$$
\left(\frac{L-3 M-6 M \omega}{L+3 M+6 M \omega}\right)^{(q+1) / 3} \equiv \omega^{i}(\bmod q) .
$$

Pro of. If $q \mid M$, it follows from Corollary 2.1 that $q^{(p-1) / 3} \equiv 1(\bmod p)$. If $q \nmid M$, using Corollary 2.1 and Theorem 2.2 we see that 


$$
\begin{aligned}
& q^{(p-1) / 3} \equiv\left(\frac{-1-L /(3 M)}{2}\right)^{i}(\bmod p) \Leftrightarrow \frac{L}{3 M} \in C_{i}(q) \\
& \Leftrightarrow\left\{\begin{array}{l}
\left(\frac{L /(3 M)-L^{\prime} /\left(3 M^{\prime}\right)}{L /(3 M)+L^{\prime} /\left(3 M^{\prime}\right)}\right)^{(q-1) / 3} \equiv\left(\frac{-1-L^{\prime} /\left(3 M^{\prime}\right)}{2}\right)^{i}(\bmod q) \\
\text { if } q \equiv 1(\bmod 3),
\end{array}\right. \\
&\left(\frac{L /(3 M)-1-2 \omega}{L /(3 M)+1+2 \omega}\right)^{(q+1) / 3} \equiv \omega^{i}(\bmod q) \quad \text { if } q \equiv 2(\bmod 3) .
\end{aligned}
$$

This completes the proof.

REMARK 2.2. In the case $i=0$ Corollary 2.2(i) was first observed by Jacobi $[\mathrm{J}]$, and Corollary 2.2(ii) can be deduced from [W1]. Inspired by K. Burde's rational biquadratic reciprocity, H. von Lienen (see [Li], [Bu]) established the first rational cubic reciprocity law.

TheOREM 2.3. Let $p>3$ be a prime, $k \in \mathbb{Z}_{p}$ and $k^{2}+3 \not \equiv 0(\bmod p)$.

(i) If $p \equiv 1(\bmod 3)$ and so $t^{2} \equiv-3(\bmod p)$ for some $t \in \mathbb{Z}$ then $k \in C_{0}(p)$ if and only if $\left(k^{2}+3\right)(k+t)$ is a cubic residue $(\bmod p)$.

(ii) If $p \equiv 2(\bmod 3)$ then $k \in C_{0}(p)$ if and only if

$$
\begin{aligned}
& \sum_{r \equiv(p+1) / 3(\bmod 3)}\left(\begin{array}{c}
(p+1) / 3 \\
r
\end{array}\right)\left(\frac{k+1}{2}\right)^{r} \\
& \equiv \frac{1}{3}\left(\frac{k+3}{2}\right)^{(p+1) / 3}+\frac{1}{3}\left(2\left(k^{2}+3\right)\right)^{-(p-2) / 3}(\bmod p) .
\end{aligned}
$$

Proof. If $p \equiv 1(\bmod 3)$, it follows from Theorem 2.2 that

$$
\begin{aligned}
k \in C_{0}(p) & \Leftrightarrow\left(\frac{k-t}{k+t}\right)^{(p-1) / 3} \equiv 1(\bmod p) \\
& \Leftrightarrow\left(\left(k^{2}+3\right)(k+t)\right)^{(p-1) / 3} \equiv 1(\bmod p) \\
& \Leftrightarrow\left(k^{2}+3\right)(k+t) \text { is a cubic residue }(\bmod p) .
\end{aligned}
$$

This proves (i).

Now consider (ii). For $i=0,1,2$ set

$$
A_{i}=\sum_{r \equiv i(\bmod 3)}\left(\begin{array}{c}
(p+1) / 3 \\
r
\end{array}\right)\left(\frac{k+1}{2}\right)^{(p+1) / 3-r} .
$$

Then $A_{0}+A_{1}+A_{2}=(1+(k+1) / 2)^{(p+1) / 3}$ and hence

$$
\left(\frac{k+1}{2}+\omega\right)^{(p+1) / 3}=A_{0}+A_{1} \omega+A_{2} \omega^{2}=A_{0}-A_{2}+\left(A_{1}-A_{2}\right) \omega
$$




$$
\begin{aligned}
= & 2 A_{0}+A_{1}-\left(\frac{k+3}{2}\right)^{(p+1) / 3} \\
& +\left(A_{0}+2 A_{1}-\left(\frac{k+3}{2}\right)^{(p+1) / 3}\right) \omega .
\end{aligned}
$$

In view of Lemma 2.2(i) we obtain

$$
\begin{aligned}
& 2^{-(p+1) / 3}\left(k^{2}+3\right)^{-(p-2) / 3}\left(\frac{k+1+2 \omega}{p}\right)_{3} \\
& \equiv 2 A_{0}+A_{1}-\left(\frac{k+3}{2}\right)^{(p+1) / 3}+\left(A_{0}+2 A_{1}-\left(\frac{k+3}{2}\right)^{(p+1) / 3}\right) \omega(\bmod p) .
\end{aligned}
$$

If $\left(\frac{k+1+2 \omega}{p}\right)_{3}=1$, it is clear that

$$
\left\{\begin{array}{l}
2 A_{0}+A_{1} \equiv\left(\frac{k+3}{2}\right)^{(p+1) / 3}+2^{-(p+1) / 3}\left(k^{2}+3\right)^{-(p-2) / 3}(\bmod p) \\
A_{0}+2 A_{1} \equiv\left(\frac{k+3}{2}\right)^{(p+1) / 3}(\bmod p)
\end{array}\right.
$$

and therefore that

$$
3 A_{0} \equiv\left(\frac{k+3}{2}\right)^{(p+1) / 3}+\left(2\left(k^{2}+3\right)\right)^{-(p-2) / 3}(\bmod p) .
$$

If $\left(\frac{k+1+2 \omega}{p}\right)_{3}=\omega$, then we have

$$
\left\{\begin{array}{l}
2 A_{0}+A_{1} \equiv\left(\frac{k+3}{2}\right)^{(p+1) / 3}(\bmod p) \\
A_{0}+2 A_{1} \equiv\left(\frac{k+3}{2}\right)^{(p+1) / 3}+2^{-(p+1) / 3}\left(k^{2}+3\right)^{-(p-2) / 3}(\bmod p)
\end{array}\right.
$$

and hence

$$
3 A_{0} \equiv\left(\frac{k+3}{2}\right)^{(p+1) / 3}-\frac{1}{2}\left(2\left(k^{2}+3\right)\right)^{-(p-2) / 3}(\bmod p) .
$$

If $\left(\frac{k+1+2 \omega}{p}\right)_{3}=\omega^{2}$, one can similarly prove that $(2.7)$ holds.

Now, by the above, (ii) follows and the proof is complete.

COROLlary 2.3. Let $m$ be the product of primes of the form $3 n+1$, and hence $t^{2} \equiv-3(\bmod m)$ for some $t \in \mathbb{Z}$. If $x \in \mathbb{Z}$ and $\left(x\left(x^{3}-1\right), m\right)=1$ then $\frac{x^{3}+1}{x^{3}-1} t \in C_{0}(m)$. 
Proof. Write $m=p_{1} \ldots p_{r}$, where $p_{1}, \ldots, p_{r}$ are primes of the form $3 n+1$. For $i=1, \ldots, r$ it is clear that $t^{2} \equiv-3\left(\bmod p_{i}\right)$. Thus,

$$
\left(\frac{x^{3}+1}{x^{3}-1} t\right)^{2}+3 \equiv 3\left(1-\left(\frac{x^{3}+1}{x^{3}-1}\right)^{2}\right) \equiv-\frac{12 x^{3}}{\left(x^{3}-1\right)^{2}} \not \equiv 0\left(\bmod p_{i}\right)
$$

and so

$$
\begin{aligned}
\left(\left(\frac{x^{3}+1}{x^{3}-1} t\right)^{2}+3\right)\left(\frac{x^{3}+1}{x^{3}-1} t+t\right) & \equiv-\frac{12 x^{3}}{\left(x^{3}-1\right)^{2}} \cdot \frac{2 x^{3} t}{x^{3}-1} \\
& \equiv\left(\frac{2 x^{2} t}{x^{3}-1}\right)^{3}\left(\bmod p_{i}\right)
\end{aligned}
$$

Applying Theorem 2.3(i) we find $\frac{x^{3}+1}{x^{3}-1} t \in C_{0}\left(p_{i}\right)$ and hence

$$
\left(\frac{\frac{x^{3}+1}{x^{3}-1} t+1+2 \omega}{m}\right)_{3}=\prod_{i=1}^{r}\left(\frac{\frac{x^{3}+1}{x^{3}-1} t+1+2 \omega}{p_{i}}\right)_{3}=1 .
$$

This is the result.

3. The structure of $C_{i}^{\prime}(p)$. In this section we introduce the sets $C_{0}^{\prime}(p), C_{1}^{\prime}(p)$ and $C_{2}^{\prime}(p)$, and study their group structure. As an application we confirm a conjecture due to K. S. Williams [W1].

Definition 3.1. Let $p \neq 3$ be a prime, $k \in \mathbb{Z}_{p},[k]_{p}=\{x \mid x \equiv k$ $\left.(\bmod p), x \in \mathbb{Z}_{p}\right\}$ and $[\infty]_{p}=\{n / m|m, n \in \mathbb{Z}, p| m, p \nmid n\}$. Define

$$
\begin{aligned}
& C_{0}^{\prime}(p)=\left\{[k]_{p} \mid k \in C_{0}(p)\right\} \cup\left\{[\infty]_{p}\right\}, \\
& C_{1}^{\prime}(p)=\left\{[k]_{p} \mid k \in C_{1}(p)\right\} \quad \text { and } \quad C_{2}^{\prime}(p)=\left\{[k]_{p} \mid k \in C_{2}(p)\right\} .
\end{aligned}
$$

As an example, taking $p=5$ we have $C_{0}^{\prime}(5)=\left\{[0]_{5},[\infty]_{5}\right\}, C_{1}^{\prime}(5)=$ $\left\{[1]_{5},[2]_{5}\right\}$ and $C_{2}^{\prime}(5)=\left\{[-1]_{5},[-2]_{5}\right\}$.

Let $p$ be a prime greater than 3 ,

$$
D_{p}= \begin{cases}\mathbb{Z} / p \mathbb{Z} & \text { if } p \equiv 1(\bmod 3), \\ \mathbb{Z}[\omega] / p \mathbb{Z}[\omega] & \text { if } p \equiv 2(\bmod 3)\end{cases}
$$

be the residue class ring modulo $p$, and $U_{p}$ the multiplicative group of $D_{p}$. It is well known that $U_{p}$ is a cyclic group of order $p^{\left(3-\left(\frac{-3}{p}\right)\right) / 2}-1$. Denote the unique subgroup of order $p-\left(\frac{-3}{p}\right)$ of $U_{p}$ by $G_{p}$. Then $G_{p}$ is also a cyclic group. So $S_{p}=\left\{\begin{array}{r}\left\{g \mid g^{p-1} \equiv 1(\bmod p), g^{n} \not \equiv 1(\bmod p)(n=1, \ldots, p-2), g \in \mathbb{Z}\right\} \\ \text { if } p \equiv 1(\bmod 3), \\ \left\{g \mid g^{p+1} \equiv 1(\bmod p), g^{n} \not \equiv 1(\bmod p)(n=1, \ldots, p), g \in \mathbb{Z}[\omega]\right\} \\ \text { if } p \equiv 2(\bmod 3)\end{array}\right.$ $\neq \emptyset$. 
We are now ready to give

TheOREM 3.1. Let $p$ be a prime greater than 3 and $g \in S_{p}$. For $i=0,1,2$ we have

$$
\left|C_{i}^{\prime}(p)\right|=\frac{p-\left(\frac{-3}{p}\right)}{3}
$$

(ii) $\quad C_{i}^{\prime}(p)$

$$
=\left\{\left[\left(\frac{-3}{p}\right)\left(1+2 g^{\left(p-\left(\frac{-3}{p}\right)\right) / 3}\right) \frac{g^{3 r+i}+1}{g^{3 r+i}-1}\right]_{p} \mid r=0,1, \ldots,\left(p-\left(\frac{-3}{p}\right)\right) / 3-1\right\} \text {. }
$$

Proof. Suppose $k \in \mathbb{Z}_{p}$ with $k^{2}+3 \not \equiv 0(\bmod p)$. If $p \equiv 1(\bmod 3)$, it is clear that $\left(-1-2 g^{(p-1) / 3}\right)^{2} \equiv-3(\bmod p)$. For $i \in\{0,1,2\}$ it follows from Theorem 2.2 that

$$
\begin{aligned}
k \in C_{i}(p) & \Leftrightarrow\left(\frac{k+1+2 g^{(p-1) / 3}}{k-1-2 g^{(p-1) / 3}}\right)^{(p-1) / 3} \equiv g^{i(p-1) / 3}(\bmod p) \\
& \Leftrightarrow \frac{k+1+2 g^{(p-1) / 3}}{k-1-2 g^{(p-1) / 3}} \equiv g^{3 r+i}(\bmod p) \\
\text { for some } r \in\{0,1, \ldots,(p-1) / 3-1\} & \Leftrightarrow k \equiv\left(1+2 g^{(p-1) / 3}\right) \frac{g^{3 r+i}+1}{g^{3 r+i}-1}(\bmod p) \\
& \text { for some } r \in\{0,1, \ldots,(p-4) / 3\} .
\end{aligned}
$$

If $p \equiv 2(\bmod 3)$, it is clear that $g^{(p+1) / 3} \equiv \omega$ or $\omega^{2}(\bmod p)$. For $i \in$ $\{0,1,2\}$ it follows from Theorem 2.2 that

$$
\begin{aligned}
k \in C_{i}(p) & \Leftrightarrow\left(\frac{k-1-2 g^{(p+1) / 3}}{k+1+2 g^{(p+1) / 3}}\right)^{(p+1) / 3} \equiv g^{i(p+1) / 3}(\bmod p) \\
& \Leftrightarrow \frac{k-1-2 g^{(p+1) / 3}}{k+1+2 g^{(p+1) / 3}} \equiv g^{3 r+i}(\bmod p) \\
\text { for some } r \in\{0,1, \ldots,(p+1) / 3-1\} & \Leftrightarrow k \equiv-\left(1+2 g^{(p+1) / 3}\right) \frac{g^{3 r+i}+1}{g^{3 r+i}-1}(\bmod p) \\
& \text { for some } r \in\{0,1, \ldots,(p-2) / 3\} .
\end{aligned}
$$

To conclude the proof, we note that

and that

$$
\left[\left(\frac{-3}{p}\right)\left(1+2 g^{\left(p-\left(\frac{-3}{p}\right)\right) / 3}\right) \frac{g^{3 \cdot 0+0}+1}{g^{3 \cdot 0+0}-1}\right]_{p}=[\infty]_{p}
$$

$$
\frac{g^{3 r_{1}+i}+1}{g^{3 r_{1}+i}-1}=1+\frac{2}{g^{3 r_{1}+i}-1} \not \equiv 1+\frac{2}{g^{3 r_{2}+i}-1}=\frac{g^{3 r_{2}+i}+1}{g^{3 r_{2}+i}-1}(\bmod p)
$$

provided $r_{1} \not \equiv r_{2}\left(\bmod \left(p-\left(\frac{-3}{p}\right)\right) / 3\right)$. 
Corollary 3.1. Let $p>3$ be a prime, and $R_{p}$ a complete residue system modulo $p$. Then

$$
\sum_{k \in C_{1}(p) \cap R_{p}} k \equiv-\frac{1}{3}(\bmod p) .
$$
that

Proof. Let $g \in S_{p}$ and $m=\left(p-\left(\frac{-3}{p}\right)\right) / 3$. It follows from Theorem 3.1

$$
\begin{aligned}
\sum_{k \in C_{1}(p) \cap R_{p}} k & \equiv\left(\frac{-3}{p}\right)\left(1+2 g^{m}\right) \sum_{r=0}^{m-1} \frac{g^{3 r+1}+1}{g^{3 r+1}-1} \\
& =\left(\frac{-3}{p}\right)\left(1+2 g^{m}\right)\left(m+\sum_{r=0}^{m-1} \frac{2}{g^{3 r+1}-1}\right)(\bmod p) .
\end{aligned}
$$

Since

$$
\begin{aligned}
\sum_{r=0}^{m-1} \frac{1}{g^{3 r+1}-1} & =\sum_{r=0}^{m-1} \frac{1}{\left(g^{3 r+1}\right)^{m}-1} \sum_{s=0}^{m-1}\left(g^{3 r+1}\right)^{s} \\
& \equiv \sum_{r=0}^{m-1} \frac{1}{g^{m}-1} \sum_{s=0}^{m-1} g^{s} \cdot g^{3 s r}=\frac{1}{g^{m}-1} \sum_{s=0}^{m-1} g^{s} \sum_{r=0}^{m-1} g^{3 s r} \\
& =\frac{1}{g^{m}-1}\left(m+\sum_{s=1}^{m-1} g^{s} \frac{1-g^{3 s m}}{1-g^{3 s}}\right) \equiv \frac{m}{g^{m}-1}(\bmod p)
\end{aligned}
$$

we find

$$
\begin{aligned}
\sum_{k \in C_{1}(p) \cap R_{p}} k & \equiv\left(\frac{-3}{p}\right)\left(1+2 g^{m}\right)\left(m+\frac{2 m}{g^{m}-1}\right) \\
& \equiv\left(\frac{-3}{p}\right) m\left(g^{m}-g^{2 m}\right) \frac{-g^{2 m}}{g^{m}-1}
\end{aligned}
$$

(Note that $1+g^{m}+g^{2 m}=\left(g^{3 m}-1\right) /\left(g^{m}-1\right) \equiv 0(\bmod p)$.)

We are done.

$$
=\left(\frac{-3}{p}\right) m g^{3 m} \equiv-\frac{1}{3}(\bmod p) .
$$

REMARK 3.1. Corollary 3.1 is equivalent to a result conjectured by K. S. Williams [W1].

Corollary 3.2. Let $p>3$ be a prime, and $R_{p}$ a complete residue system modulo $p$. Then

$$
\begin{aligned}
& \mid\left\{k \mid k \in C_{1}(p)\right.\left.\cap R_{p},\left(\frac{k^{2}+3}{p}\right)=1\right\} \mid \\
&=\left|\left\{k \mid k \in C_{1}(p) \cap R_{p},\left(\frac{k^{2}+3}{p}\right)=-1\right\}\right|=\frac{p-\left(\frac{-3}{p}\right)}{6} .
\end{aligned}
$$


Proof. Let $g \in S_{p}$. In view of Theorem 3.1 we can write

$$
C_{1}(p) \cap R_{p}=\left\{k_{r} \mid r=0,1, \ldots,\left(p-\left(\frac{-3}{p}\right)\right) / 3-1\right\},
$$

where

$$
k_{r} \equiv\left(\frac{-3}{p}\right)\left(1+2 g^{\left(p-\left(\frac{-3}{p}\right)\right) / 3}\right) \frac{g^{3 r+1}+1}{g^{3 r+1}-1}(\bmod p) .
$$

From this it follows that

$$
\begin{aligned}
k_{r}^{2} & \equiv\left(1+4 g^{\left(p-\left(\frac{-3}{p}\right)\right) / 3}+4 g^{\left(2\left(p-\left(\frac{-3}{p}\right)\right)\right) / 3}\right)\left(1+\frac{2}{g^{3 r+1}-1}\right)^{2} \\
& \equiv-3\left(1+\frac{2}{g^{3 r+1}-1}\left(2+\frac{2}{g^{3 r+1}-1}\right)\right)=-3-\frac{3 \cdot 4 \cdot g^{3 r+1}}{\left(g^{3 r+1}-1\right)^{2}}(\bmod p)
\end{aligned}
$$

and so

$$
\begin{aligned}
\left(\frac{k_{r}^{2}+3}{p}\right) & \equiv\left(k_{r}^{2}+3\right)^{(p-1) / 2} \equiv(-3 \cdot 4)^{(p-1) / 2} \cdot g^{\frac{p-1}{2}(3 r+1)} \cdot \frac{g^{3 r+1}-1}{\left(g^{3 r+1}-1\right)^{p}} \\
& \equiv\left(\frac{-3}{p}\right) g^{\frac{p-\left(\frac{-3}{p}\right)}{2}(3 r+1)} \cdot g^{\frac{\left(\frac{-3}{p}\right)-1}{2}(3 r+1)} \cdot \frac{g^{3 r+1}-1}{g^{(3 r+1) p}-1} \\
& \equiv\left(\frac{-3}{p}\right)(-1)^{3 r+1} g^{\frac{\left(\frac{-3}{p}\right)-1}{2}(3 r+1)} \frac{g^{3 r+1}-1}{g^{\left(\frac{-3}{p}\right)(3 r+1)}-1} \\
& =(-1)^{r+1}(\bmod p) .
\end{aligned}
$$

Thus,

$$
\left(\frac{k_{2 n}^{2}+3}{p}\right)=-\left(\frac{k_{2 n+1}^{2}+3}{p}\right)=-1 \quad \text { for } n=0,1, \ldots,\left(p-\left(\frac{-3}{p}\right)\right) / 6-1 .
$$

This proves the corollary.

THEOREM 3.2. Let $p$ be a prime greater than 3 . For $[k]_{p},\left[k^{\prime}\right]_{p} \in C_{0}^{\prime}(p) \cup$ $C_{1}^{\prime}(p) \cup C_{2}^{\prime}(p)$ define

$$
[k]_{p} *\left[k^{\prime}\right]_{p}=\left[\frac{k k^{\prime}-3}{k+k^{\prime}}\right]_{p} \quad\left([k]_{p} *[\infty]_{p}=[\infty]_{p} *[k]_{p}=[k]_{p}\right) .
$$

Then $C_{0}^{\prime}(p) \cup C_{1}^{\prime}(p) \cup C_{2}^{\prime}(p)$ forms a cyclic group of order $p-\left(\frac{-3}{p}\right)$, and $C_{0}^{\prime}(p)$ is a subgroup of order $\left(p-\left(\frac{-3}{p}\right)\right) / 3$. Moreover, $C_{0}^{\prime}(p), C_{1}^{\prime}(p)$ and $C_{2}^{\prime}(p)$ are the three distinct cosets of $C_{0}^{\prime}(p)$.

Proof. Suppose $g \in S_{p}$. From Theorem 3.1 we know that

$$
C_{0}^{\prime}(p) \cup C_{1}^{\prime}(p) \cup C_{2}^{\prime}(p)=\left\{\left[k_{r}\right]_{p} \mid r=0,1, \ldots, p-\left(\frac{-3}{p}\right)-1\right\},
$$

where

$$
\left[k_{r}\right]_{p}=\left[\left(\frac{-3}{p}\right)\left(1+2 g^{\left(p-\left(\frac{-3}{p}\right)\right) / 3}\right) \frac{g^{r}+1}{g^{r}-1}\right]_{p}
$$


Since

$$
\begin{aligned}
& {\left[\frac{k_{i} k_{j}-3}{k_{i}+k_{j}}\right]_{p}=\left[\frac{\left(\left(\frac{-3}{p}\right)\left(1+2 g^{\left(p-\left(\frac{-3}{p}\right)\right) / 3}\right)\right)^{2} \cdot \frac{g^{i}+1}{g^{i}-1} \cdot \frac{g^{j}+1}{g^{j}-1}-3}{\left(\frac{-3}{p}\right)\left(1+2 g^{\left(p-\left(\frac{-3}{p}\right)\right) / 3}\right)\left(\frac{g^{i}+1}{g^{i}-1}+\frac{g^{j}+1}{g^{j}-1}\right)}\right]_{p}} \\
& =\left[\left(\frac{-3}{p}\right)\left(1+2 g^{\left(p-\left(\frac{-3}{p}\right)\right) / 3}\right) \frac{\left(g^{i}+1\right)\left(g^{j}+1\right)+\left(g^{i}-1\right)\left(g^{j}-1\right)}{\left(g^{i}+1\right)\left(g^{j}-1\right)+\left(g^{i}-1\right)\left(g^{j}+1\right)}\right]_{p} \\
& =\left[\left(\frac{-3}{p}\right)\left(1+2 g^{\left(p-\left(\frac{-3}{p}\right)\right) / 3}\right) \frac{g^{i+j}+1}{g^{i+j}-1}\right]_{p},
\end{aligned}
$$

we see that

$$
\left[k_{i}\right]_{p} *\left[k_{j}\right]_{p}=\left[\frac{k_{i} k_{j}-3}{k_{i}+k_{j}}\right]_{p}=\left[k_{\langle i+j\rangle}\right]_{p},
$$

where $\langle x\rangle$ denotes the least nonnegative residue of $x$ modulo $p-\left(\frac{-3}{p}\right)$.

By the above, $C_{0}^{\prime}(p) \cup C_{1}^{\prime}(p) \cup C_{2}^{\prime}(p)$ is a cyclic group generated by $\left[k_{1}\right]_{p}$. Applying Theorem 3.1 we see that $C_{0}^{\prime}(p)$ is a cyclic group generated by $\left[k_{3}\right]_{p}$, and that $C_{0}^{\prime}(p), C_{1}^{\prime}(p)$ and $C_{2}^{\prime}(p)$ are the three cosets of $C_{0}^{\prime}(p)$. The proof is now complete.

Corollary 3.3. Let $p$ be a prime greater than 3 . Then

$$
C_{0}^{\prime}(p)=\left\{\left[\frac{x^{3}-9 x}{3 x^{2}-3}\right]_{p} \mid x \in\{0,1, \ldots, p-1\}, x^{2} \not \equiv-3(\bmod p)\right\} .
$$

Proof. Clearly

$$
\left[\frac{1^{3}-9 \cdot 1}{3 \cdot 1^{2}-3}\right]_{p}=[\infty]_{p} \in C_{0}^{\prime}(p)
$$

Suppose $k \in \mathbb{Z}_{p}$. It follows from Theorem 3.2 that

$$
\begin{aligned}
{[k]_{p} \in C_{0}^{\prime}(p) \Leftrightarrow } & {[k]_{p}=[x]_{p} *[x]_{p} *[x]_{p} } \\
& \text { for some }[x]_{p} \in C_{0}^{\prime}(p) \cup C_{1}^{\prime}(p) \cup C_{2}^{\prime}(p) \\
\Leftrightarrow & {[k]_{p}=\left[\frac{x^{2}-3}{2 x}\right]_{p} *[x]_{p}=\left[\frac{x^{3}-9 x}{3 x^{2}-3}\right]_{p} \text { for some integer } x } \\
& \text { satisfying } x^{2}+3 \not \equiv 0(\bmod p) \text { and } x \in\{0,1, \ldots, p-1\}
\end{aligned}
$$

So the result follows.

Corollary 3.4. Let $p>3$ be a prime, $i \in\{0,1,2\}$ and $\left[k_{i}\right]_{p} \in C_{i}^{\prime}(p)$. For $[k]_{p} \in C_{0}^{\prime}(p)$ define

$$
\varphi\left([k]_{p}\right)=\left[\frac{k k_{i}-3}{k+k_{i}}\right]_{p} \quad\left(\varphi\left([\infty]_{p}\right)=\left[k_{i}\right]_{p}\right) .
$$

Then $\varphi$ is a one-to-one correspondence from $C_{0}^{\prime}(p)$ to $C_{i}^{\prime}(p)$. 
Proof. In view of Theorem 3.2,

So the result follows.

$$
C_{i}^{\prime}(p)=\left[k_{i}\right]_{p} C_{0}^{\prime}(p)=\left\{\varphi\left([k]_{p}\right) \mid[k]_{p} \in C_{0}^{\prime}(p)\right\} .
$$

REMARK 3.2. Corollaries 3.3 and 3.4 provide a simple method of calculating $C_{0}^{\prime}(p), C_{1}^{\prime}(p)$ and $C_{2}^{\prime}(p)$ for any prime $p>3$.

4. Cubic congruences. Let $p$ be a prime greater than 3 . In this section we consider the general cubic congruence $x^{3}+A x^{2}+B x+C \equiv 0$ $(\bmod p)$, where $A, B, C \in \mathbb{Z}_{p}$.

In [St] Stickelberger showed that the number of solutions of $x^{3}+A x^{2}+$ $B x+C \equiv 0(\bmod p)$ is given by

$$
N= \begin{cases}0 \text { or } 3 & \text { if }\left(\frac{D}{p}\right)=1, \\ 1 & \text { if }\left(\frac{D}{p}\right)=-1,\end{cases}
$$

where $D=A^{2} B^{2}-4 B^{3}-4 A^{3} C-27 C^{2}+18 A B C$.

Since

$x^{3}+A x^{2}+B x+C=\left(x+\frac{A}{3}\right)^{3}-3 \cdot \frac{A^{2}-3 B}{9}\left(x+\frac{A}{3}\right)+\frac{2 A^{3}-9 A B+27 C}{27}$, it is enough to discuss the congruence $x^{3}-3 a x-a b \equiv 0(\bmod p)\left(a, b \in \mathbb{Z}_{p}\right)$.

LEMMA 4.1. Assume that $p$ is a prime greater than $3, k \in \mathbb{Z}_{p}$ and $k^{2}+3 \not \equiv 0(\bmod p)$. Then $k \in C_{0}(p)$ if and only if the congruence $x^{3}-$ $9\left(k^{2}+3\right) x-18\left(k^{2}+3\right) \equiv 0(\bmod p)$ is solvable. Moreover, if $k \in C_{0}(p)$ then the solutions of the above congruence are given by

$$
x \equiv \begin{cases}(-3+k t) u(1-u)(\bmod p) & \text { if } p \equiv 1(\bmod 3) \\ (k-3+2 k \omega) u(1-u)(\bmod p) & \text { if } p \equiv 2(\bmod 3),\end{cases}
$$

where $t$ and $u$ satisfy $t^{2} \equiv-3(\bmod p)(t \in \mathbb{Z})$ and

$$
u^{3} \equiv \begin{cases}\frac{k-t}{k+t}(\bmod p)(u \in \mathbb{Z}) & \text { if } p \equiv 1(\bmod 3), \\ \frac{k-1-2 \omega}{k+1+2 \omega}(\bmod p)(u \in \mathbb{Z}[\omega]) & \text { if } p \equiv 2(\bmod 3) .\end{cases}
$$

Proof. If $k \equiv 0(\bmod p)$, then $k \in C_{0}(p)$. Clearly, the congruence $x^{3}-9\left(k^{2}+3\right) x-18\left(k^{2}+3\right) \equiv 0(\bmod p)$ has the solutions $x \equiv 6,-3,-3$ $(\bmod p)$. So the result is true when $p \mid k$.

Now assume $k \not \equiv 0(\bmod p)$. It follows from Corollary 3.3 that

$$
\begin{aligned}
k \in C_{0}(p) & \Leftrightarrow k \equiv \frac{s^{3}-9 s}{3 s^{2}-3}(\bmod p) \text { for some } s \in\{0,1, \ldots, p-1\} \\
& \Leftrightarrow s^{3}-3 k s^{2}-9 s+3 k \equiv 0(\bmod p) \text { is solvable. }
\end{aligned}
$$


Set $x \equiv \frac{3(k-s)}{s}(\bmod p)$. Then

$$
\begin{aligned}
\frac{9 k^{2}}{s^{3}}\left(s^{3}-3 k s^{2}-9 s+3 k\right) & =9 k^{2}-9 k^{2} \cdot \frac{3 k}{s}-9\left(\frac{3 k}{s}\right)^{2}+\left(\frac{3 k}{s}\right)^{3} \\
& \equiv 9 k^{2}-9 k^{2}(x+3)-9(x+3)^{2}+(x+3)^{3} \\
& =x^{3}-9\left(k^{2}+3\right) x-18\left(k^{2}+3\right)(\bmod p) .
\end{aligned}
$$

So $k \in C_{0}(p)$ if and only if $x^{3}-9\left(k^{2}+3\right) x-18\left(k^{2}+3\right) \equiv 0(\bmod p)$ is solvable.

Let $k \in C_{0}(p)$ and $r=t$ or $1+2 \omega$ according as $p \equiv 1$ or $2(\bmod 3)$. From Theorem 2.2 we know that

So the congruence

$$
\left(\frac{k-r}{k+r}\right)^{\left(p-\left(\frac{-3}{p}\right)\right) / 3} \equiv 1(\bmod p) .
$$

$$
u^{3} \equiv \frac{k-r}{k+r}(\bmod p)
$$

is solvable. Suppose $u^{3} \equiv \frac{k-r}{k+r}(\bmod p)$ and $x \equiv(-3+k r) u(1-u)(\bmod p)$. Then

$$
\begin{aligned}
u^{3}(1-u)^{3} & =u^{3}\left(1-3 u+3 u^{2}-u^{3}\right) \\
& \equiv \frac{k-r}{k+r}\left(\frac{2 r}{k+r}-3 u+3 u^{2}\right)(\bmod p)
\end{aligned}
$$

and hence

$$
\begin{aligned}
x^{3}-9\left(k^{2}+3\right) x \equiv & (-3+k r)^{3} u^{3}(1-u)^{3}-9\left(k^{2}+3\right)(-3+k r)\left(u-u^{2}\right) \\
\equiv & r^{3}(k+r)^{3} \frac{k-r}{k+r}\left(\frac{2 r}{k+r}-3 u+3 u^{2}\right) \\
& -9 r(k+r)^{2}(k-r)\left(u-u^{2}\right) \\
\equiv & 18\left(k^{2}+3\right)(\bmod p) .
\end{aligned}
$$

When $p \equiv 2(\bmod 3)$ it is easily seen that $u^{3} \bar{u}^{3} \equiv 1(\bmod p)$ and so that $\bar{u} \equiv u^{-1}(\bmod p)$. Hence,

$$
\begin{aligned}
\overline{(k-3+2 k \omega) u(1-u)} & =\overline{(1+2 \omega)} \overline{(k+1+2 \omega)} \bar{u}(1-\bar{u}) \\
& \equiv(-1-2 \omega)(k-1-2 \omega) \frac{1}{u}\left(1-\frac{1}{u}\right) \\
& =(1+2 \omega)(k-1-2 \omega) \frac{u(1-u)}{u^{3}} \\
& \equiv(k-3+2 k \omega) u(1-u)(\bmod p) .
\end{aligned}
$$

This shows that $(k-3+2 k \omega) u(1-u)$ is congruent to an integer modulo $p$.

By the above, the lemma is proved. 
THEOREM 4.1. Let $p>3$ be a prime, $a, b, s \in \mathbb{Z}_{p}, a b \not \equiv 0(\bmod p)$ and $s^{2} \equiv-3\left(b^{2}-4 a\right)(\bmod p)$. Then the congruence $x^{3}-3 a x-a b \equiv 0(\bmod p)$ is solvable if and only if $s / b \in C_{0}(p)$. Moreover, if $s / b \in C_{0}(p)$ then the solutions of the above congruence are given by

$$
x \equiv \begin{cases}\frac{1}{6}(s t-3 b) u(1-u)(\bmod p) & \text { if } p \equiv 1(\bmod 3), \\ \frac{1}{6}(s-3 b+2 s \omega) u(1-u)(\bmod p) & \text { if } p \equiv 2(\bmod 3),\end{cases}
$$

where $t$ and $u$ satisfy $t^{2} \equiv-3(\bmod p)(t \in \mathbb{Z})$ and

$$
u^{3} \equiv \begin{cases}\frac{s-b t}{s+b t}(\bmod p)(u \in \mathbb{Z}) & \text { if } p \equiv 1(\bmod 3), \\ \frac{s-b(1+2 \omega)}{s+b(1+2 \omega)}(\bmod p)(u \in \mathbb{Z}[\omega]) & \text { if } p \equiv 2(\bmod 3)\end{cases}
$$

Proof. Set $k=s / b$. Then $k^{2}+3 \equiv 12 a / b^{2} \not \equiv 0(\bmod p)$. It is clear that

$$
\begin{aligned}
x^{3}-9\left(k^{2}+3\right) x-18\left(k^{2}+3\right) & \equiv x^{3}-\frac{108 a}{b^{2}} x-\frac{216 a}{b^{2}} \\
& =\left(\frac{6}{b}\right)^{3}\left(\left(\frac{b}{6} x\right)^{3}-3 a \cdot \frac{b}{6} x-a b\right)(\bmod p) .
\end{aligned}
$$

So the result follows from Lemma 4.1.

Corollary 4.1. Let $p>3$ be a prime and $a, b \in \mathbb{Z}_{p}$. Then the congruence $x^{3}-3 a x-a b \equiv 0(\bmod p)$ is unsolvable if and only if $-3\left(b^{2}-4 a\right) \equiv k^{2} b^{2}$ $(\bmod p)$ for some $k \in C_{1}(p)$.

Proof. If $a b \equiv 0(\bmod p)$ then $0^{3}-3 a \cdot 0-a b \equiv 0(\bmod p)$. If $b^{2}-4 a \equiv 0$ $(\bmod p)$ then $b^{3}-3 a b-a b \equiv 0(\bmod p)$. So $x^{3}-3 a x-a b \equiv 0(\bmod p)$ is solvable when $a b\left(b^{2}-4 a\right) \equiv 0(\bmod p)$.

Now assume $a b\left(b^{2}-4 a\right) \not \equiv 0(\bmod p)$. Since $-4(-3 a)^{3}-27(-a b)^{2}=$ $-3\left(b^{2}-4 a\right) \cdot 9 a^{2}$, using Stickelberger's result we see that $x^{3}-3 a x-a b \equiv 0$ $(\bmod p)$ has one solution if $\left(\frac{-3\left(b^{2}-4 a\right)}{p}\right)=-1$.

If $\left(\frac{-3\left(b^{2}-4 a\right)}{p}\right)=1$, there is an integer $k$ such that $k^{2} \equiv-3\left(b^{2}-4 a\right) / b^{2}$ $(\bmod p)$. Since $k^{2}+3 \equiv 12 a / b^{2} \not \equiv 0(\bmod p)$ we have $k \in C_{0}(p) \cup C_{1}(p) \cup$ $C_{2}(p)$. Applying Theorem 4.1 we see that $x^{3}-3 a x-a b \equiv 0(\bmod p)$ is solvable if and only if $k \in C_{0}(p)$. So $x^{3}-3 a x-a b \equiv 0(\bmod p)$ is unsolvable if and only if $-3\left(b^{2}-4 a\right) \equiv k^{2} b^{2}(\bmod p)$ for some $k \in C_{1}(p) \cup C_{2}(p)$.

Since $k \in C_{2}(p)$ if and only if $-k \in C_{1}(p)$, by the above the corollary is proved.

REMARK 4.1. If $p$ is a prime greater than $3, a, b \in \mathbb{Z}_{p}$ and $\left(\frac{-3\left(b^{2}-4 a\right)}{p}\right)=$ -1 , one can easily check that the unique solution of $x^{3}-3 a x-a b \equiv 0(\bmod p)$ is given by 


$$
x \equiv a^{\left(p-\left(\frac{-3}{p}\right)\right) / 3} v_{\left(p+2\left(\frac{-3}{p}\right)\right) / 3}(\bmod p),
$$

where $\left\{v_{n}\right\}$ is defined by $v_{0}=2, v_{1}=b$ and $v_{n+1}=b v_{n}-a v_{n-1}(n \geq 1)$.

As applications of Theorem 4.1 we have

Theorem 4.2. Let $p>3$ be a prime, $m, n \in \mathbb{Z}_{p}$ and $m n \not \equiv 0(\bmod p)$. Then

$$
\left|\left\{\left[\frac{x^{3}}{m x+n}\right]_{p} \mid x \neq-\frac{n}{m}(\bmod p), x \in \mathbb{Z}_{p}\right\}\right|=p-\frac{p-\left(\frac{-3}{p}\right)}{3} .
$$

Proof. Set $b=3 n / m$. Then $b \in \mathbb{Z}_{p}$ and $b \not \equiv 0(\bmod p)$. From Corollary 4.1 and Theorem 3.1(i) we see that

$$
\begin{aligned}
& \left|\left\{\left[\frac{x^{3}}{m x+n}\right]_{p} \mid x \in \mathbb{Z}_{p}, x \neq \equiv-\frac{n}{m}(\bmod p)\right\}\right| \\
& \quad=\left|\left\{\left[\frac{x^{3}}{3 x+b}\right]_{p} \mid x \in \mathbb{Z}_{p}, x \neq \equiv-\frac{b}{3}(\bmod p)\right\}\right| \\
& \quad=\mid\left\{[a]_{p} \mid x^{3}-3 a x-a b \equiv 0(\bmod p) \text { is solvable }\right\} \mid \\
& \quad=p-\mid\left\{[a]_{p} \mid x^{3}-3 a x-a b \equiv 0(\bmod p) \text { is unsolvable }\right\} \mid \\
& \quad=p-\mid\left\{[a]_{p} \mid-3\left(b^{2}-4 a\right) \equiv k^{2} b^{2}(\bmod p) \text { for some } k \in C_{1}(p)\right\} \mid \\
& \quad=p-\left|\left\{\left[\frac{\left(k^{2}+3\right) b^{2}}{12}\right]_{p} \mid k \in C_{1}(p)\right\}\right|=p-\frac{p-\left(\frac{-3}{p}\right)}{3} .
\end{aligned}
$$

We are done.

Theorem 4.3. Let $p>3$ be a prime and $A, B, C \in \mathbb{Z}_{p}$. Then

$$
\begin{aligned}
& \left|\left\{\left[x^{3}+A x^{2}+B x+C\right]_{p} \mid x \in \mathbb{Z}_{p}\right\}\right| \\
& =\left|\left\{\left[x^{3}+A x^{2}+B x+C\right]_{p} \mid x \in\{0,1, \ldots, p-1\}\right\}\right| \\
& = \begin{cases}\frac{p+2}{3} & \text { if } p \equiv 1(\bmod 3) \text { and } A^{2} \equiv 3 B(\bmod p), \\
p & \text { if } p \equiv 2(\bmod 3) \text { and } A^{2} \equiv 3 B(\bmod p), \\
p-\frac{p-\left(\frac{-3}{p}\right)}{3} & \text { if } A^{2} \not \equiv 3 B(\bmod p) .\end{cases}
\end{aligned}
$$

Proof. Since

$x^{3}+A x^{2}+B x+C=\left(x+\frac{A}{3}\right)^{3}-\frac{A^{2}-3 B}{3}\left(x+\frac{A}{3}\right)+\frac{2 A^{3}-9 A B+27 C}{27}$

we see that 


$$
\begin{aligned}
& \left|\left\{\left[x^{3}+A x^{2}+B x+C\right]_{p} \mid x \in \mathbb{Z}_{p}\right\}\right| \\
& =\left|\left\{\left[x^{3}-\frac{A^{2}-3 B}{3} x+\frac{2 A^{3}-9 A B+27 C}{27}\right]_{p} \mid x \in \mathbb{Z}_{p}\right\}\right| \\
& =\left|\left\{\left[x^{3}-\frac{A^{2}-3 B}{3} x\right]_{p} \mid x \in \mathbb{Z}_{p}\right\}\right| \\
& =\mid\left\{[t]_{p} \mid x^{3}-\frac{A^{2}-3 B}{3} x \equiv t(\bmod p) \text { is solvable, } t \in \mathbb{Z}_{p}\right\} \mid \\
& = \begin{cases}1+\frac{p-1}{3} & \text { if } p \equiv 1(\bmod 3) \text { and } A^{2} \equiv 3 B(\bmod p), \\
p & \text { if } p \equiv 2(\bmod 3) \text { and } A^{2} \equiv 3 B(\bmod p), \\
\mid\left\{[b]_{p} \mid x^{3}-3 \cdot \frac{A^{2}-3 B}{9} x-\frac{A^{2}-3 B}{9} b \equiv 0(\bmod p) \text { is solvable }\right\} \mid & \text { if } A^{2} \not \equiv 3 B(\bmod p) .\end{cases}
\end{aligned}
$$

Now suppose $A^{2} \not \equiv 3 B(\bmod p)$ and $a=\left(A^{2}-3 B\right) / 9$. By Corollaries 4.1 and 3.2 we get

$$
\begin{aligned}
\mid\left\{[b]_{p}\right. & \left.\mid x^{3}-3 a x-a b \equiv 0(\bmod p) \text { is solvable }\right\} \mid \\
& =p-\mid\left\{[b]_{p} \mid x^{3}-3 a x-a b \equiv 0(\bmod p) \text { is unsolvable }\right\} \mid \\
& =p-\mid\left\{[b]_{p} \mid-3\left(b^{2}-4 a\right) \equiv k^{2} b^{2}(\bmod p) \text { for some } k \in C_{1}(p)\right\} \mid \\
& =p-\mid\left\{[b]_{p} \mid b^{2} \equiv \frac{12 a}{k^{2}+3}(\bmod p) \text { for some } k \in C_{1}(p)\right\} \mid \\
& =p-2\left|\left\{\left[\frac{12 a}{k^{2}+3}\right]_{p} \mid\left(\frac{k^{2}+3}{p}\right)=\left(\frac{12 a}{p}\right), k \in C_{1}(p)\right\}\right| \\
& =p-2 \cdot \frac{p-\left(\frac{-3}{p}\right)}{6} .
\end{aligned}
$$

Putting the above together yields the result.

5. Connections with binary quadratic forms. Let $d$ be a squarefree integer, and $p$ a prime greater than 3 satisfying $\left(\frac{d}{p}\right)=1$. In this section we obtain a criterion for $s(d) \in C_{i}(p)(i \in\{0,1,2\})$ in terms of the binary quadratic forms of discriminant $4 d$, where $s(d)$ satisfies $(s(d))^{2} \equiv d(\bmod p)$.

THEOREM 5.1. Let $p$ be a prime greater than 3 and $p=a x^{2}+2 b x y+c y^{2}$ with $a, b, c, x, y \in \mathbb{Z}$. If $d=b^{2}-a c, a=2^{\alpha} 3^{r} a_{1}\left(2 \nmid a_{1}, 3 \nmid a_{1}\right), d+3=2^{\beta} 3^{s} d_{1}$ $\left(2 \nmid d_{1}, 3 \nmid d_{1}\right),(a, d+3)=1$ and $a(d+3) \not \equiv 0(\bmod p)$, then 


$$
\begin{aligned}
& \left(\frac{a x+(b+1) y+2 y \omega}{a_{1} d_{1}^{2} p}\right)_{3} \\
& = \begin{cases}\omega^{(1-s)\left(\frac{-3}{a x}\right) \frac{y}{3}} & \text { if } y \equiv 0(\bmod 3), \\
\omega^{f_{1}(u)} & \text { if } a \equiv 0(\bmod 3) \text { and } x \equiv u y(\bmod 9), \\
\omega^{f_{2}(u)} & \text { if } a(a x+b y) \not \equiv 0(\bmod 3) \text { and } x \equiv u y(\bmod 9), \\
1 & \text { if } a x+b y \equiv 0(\bmod 9), \\
\omega^{ \pm\left(\frac{-3}{d}\right)} & \text { if } a x+b y \equiv \pm 3 y(\bmod 9),\end{cases}
\end{aligned}
$$

where

$$
\begin{aligned}
f_{1}(u)= & \frac{1}{3}\left(\frac{-3}{b}\right)\left((2 b u+c)\left(\frac{-3}{2 b u+c}\right)-1\right. \\
& \left.+(r-1)\left(1-b\left(\frac{-3}{b}\right)\right)+a c+2^{\alpha} a_{1}\left(\frac{-3}{2^{\alpha} a_{1}}\right)-4\right), \\
f_{2}(u)= & \frac{1}{3}\left(\frac{-3}{a u+b}\right)\left(\left(s-\left(\frac{-3}{1-d}\right)\right)\left((a u+b)\left(\frac{-3}{a u+b}\right)-1\right)\right. \\
& \left.+(1-d)\left(\frac{-3}{1-d}\right)-2^{\beta} d_{1}\left(\frac{-3}{2^{\beta} d_{1}}\right)\right)
\end{aligned}
$$

and

$$
\left(\frac{-3}{m}\right)= \begin{cases}1 & \text { if } m \equiv 1(\bmod 3) \\ -1 & \text { if } m \equiv-1(\bmod 3)\end{cases}
$$

Proof. For later use we first point out the following facts:

(i) $y \not \equiv 0(\bmod p)$. Indeed, if $p \mid y$ then $p \mid a x^{2}$ and so $p \mid x$. Thus, $p=a x^{2}+2 b x y+c y^{2} \equiv 0\left(\bmod p^{2}\right)$. This is a contradiction.

(ii) If $\pi=a x+b y+y+2 y \omega$ then $(N \pi, a)=(N \pi, d+3)=1$. Indeed, clearly $N \pi=\pi \bar{\pi}=(a x+b y)^{2}+3 y^{2}=a p+(d+3) y^{2}$. Thus, $(N \pi, a)=$ $\left((d+3) y^{2}, a\right)=1$ and $(N \pi, d+3)=(a p, d+3)=1$.

(iii) If $A+B \omega \in \mathbb{Z}[\omega]$ and $A+B \omega \equiv 2(\bmod 3)$ then $\left(\frac{3}{A+B \omega}\right)_{3}=\omega^{-B / 3}$. Indeed, since $3=-\omega^{2}(1-\omega)^{2}$, it follows from (1.1) and (1.2) that

$$
\begin{aligned}
\left(\frac{3}{A+B \omega}\right)_{3} & =\left(\frac{\omega}{A+B \omega}\right)_{3}^{2}\left(\frac{1-\omega}{A+B \omega}\right)_{3}^{2} \\
& =\omega^{2(A+B+1) / 3} \cdot \omega^{4(A+1) / 3}=\omega^{-B / 3} .
\end{aligned}
$$

Now let $\pi=a x+(b+1) y+2 y \omega$. Since $N(1-\omega)=3$ and $N \pi=$ $(a x+b y)^{2}+3 y^{2} \not \equiv 0(\bmod 9)$, there are integers $i, k \in\{0,1\}$ and $j \in\{0,1,2\}$ such that $\pi=(-1)^{i} \omega^{j}(1-\omega)^{k} \pi^{\prime}$, where $\pi^{\prime} \in \mathbb{Z}[\omega]$ and $\pi^{\prime} \equiv 2(\bmod 3)$. 
Assume $y=3^{t} y_{0}\left(3 \nmid y_{0}\right)$ and $\pi^{\prime}=A+B \omega$. Then we have

$$
\begin{aligned}
& \left(\frac{a x+(b+1) y+2 y \omega}{p}\right)_{3}=\left(\frac{(-1)^{i} \omega^{j}(1-\omega)^{k} \pi^{\prime}}{p}\right)_{3} \\
& =\left(\frac{\omega}{p}\right)_{3}^{j-k}\left(\frac{\omega(1-\omega)}{p}\right)_{3}^{k}\left(\frac{\pi^{\prime}}{p}\right)_{3}=\left(\frac{\omega}{p}\right)_{3}^{j-k}\left(\frac{1+2 \omega}{p}\right)_{3}^{k}\left(\frac{p}{\pi^{\prime}}\right)_{3} \\
& =\left(\frac{\omega}{p}\right)_{3}^{j-k}\left(\frac{a p}{\pi^{\prime}}\right)_{3}\left(\frac{a^{2}}{\pi^{\prime}}\right)_{3}=\left(\frac{\omega}{p}\right)_{3}^{j-k}\left(\frac{-(d+3) y^{2}}{\pi^{\prime}}\right)_{3}\left(\frac{a^{2}}{\pi^{\prime}}\right)_{3} \\
& =\left(\frac{\omega}{p}\right)_{3}^{j-k}\left(\frac{3^{2 r+s+2 t}}{\pi^{\prime}}\right)_{3}\left(\frac{2^{2 \alpha+\beta} a_{1}^{2} d_{1} y_{0}^{2}}{\pi^{\prime}}\right)_{3} \\
& =\left(\frac{\omega}{p}\right)_{3}^{j-k}\left(\frac{3}{\pi^{\prime}}\right)_{3}^{s-r-t}\left(\frac{\pi^{\prime}}{2^{2 \alpha+\beta} a_{1}^{2} d_{1} y_{0}^{2}}\right)_{3} \\
& =\left(\frac{\omega}{p}\right)_{3}^{j-k} \omega^{-(s-r-t) B / 3}\left(\frac{\pi}{2^{2 \alpha+\beta} a_{1}^{2} d_{1} y_{0}^{2}}\right)_{3}\left(\frac{(-1)^{i} \omega^{j-k}(1+2 \omega)^{k}}{2^{2 \alpha+\beta} a_{1}^{2} d_{1} y_{0}^{2}}\right)_{3}^{-1} \\
& =\left(\frac{\omega}{p}\right)_{3}^{j-k} \omega^{(r+t-s) B / 3}\left(\frac{\omega}{2^{2 \alpha+\beta} a_{1}^{2} d_{1} y_{0}^{2}}\right)_{3}^{k-j}\left(\frac{\pi}{a_{1}^{2} d_{1}}\right)_{3}\left(\frac{\pi}{2}\right)_{3}^{2 \alpha+\beta}\left(\frac{\pi}{y_{0}}\right)_{3}^{2} \\
& \text { (by Proposition 2.1) } \\
& =\left(\frac{\omega}{p}\right)_{3}^{j-k} \omega^{(r+t-s) B / 3}\left(\frac{\omega}{2^{2 \alpha+\beta} a_{1}^{2} d_{1} y_{0}^{2}}\right)_{3}^{2(j-k)}\left(\frac{\pi}{a_{1}^{2} d_{1}}\right)_{3} \\
& \text { (Note that }\left(\frac{\pi}{2}\right)_{3}=\left(\frac{\pi}{y_{0}}\right)_{3}=1 \text {.) } \\
& =\left(\frac{\omega}{2^{\alpha+2 \beta} a_{1} d_{1}^{2} p y_{0}}\right)_{3}^{j-k} \omega^{(r+t-s) B / 3}\left(\frac{\pi}{a_{1} d_{1}^{2}}\right)_{3}^{-1} .
\end{aligned}
$$

That is,

$$
\left(\frac{a x+(b+1) y+2 y \omega}{a_{1} d_{1}^{2} p}\right)_{3}=\omega^{(r+t-s) B / 3}\left(\frac{\omega}{2^{\alpha+2 \beta} a_{1} d_{1}^{2} p y_{0}}\right)_{3}^{j-k} .
$$

Let us consider five cases.

CASE 1: $y \equiv 0(\bmod 3)$. Since $p=a x^{2}+2 b x y+c y^{2} \equiv a x^{2}(\bmod 3)$ we have $a x \not \equiv 0(\bmod 3)$ and so $r=0$. Clearly $\pi^{\prime}=-\left(\frac{-3}{a x}\right) \pi$. Hence $j=k=0$ and $B=-2\left(\frac{-3}{a x}\right) y$. From (5.1) we see that

$$
\left(\frac{a x+(b+1) y+2 y \omega}{a_{1} d_{1}^{2} p}\right)_{3}=\omega^{-\left(\frac{-3}{a x}\right) \frac{2 y}{3}(t-s)}=\omega^{(1-s)\left(\frac{-3}{a x}\right) \frac{y}{3}} .
$$

CASE $2: a \equiv 0(\bmod 3)$. In this case, $y \not \equiv 0(\bmod 3)$. Since $(a, d+3)=1$ we have $b^{2}=a c-3+(d+3) \not \equiv 0(\bmod 3)$ and so $a x+b y \not \equiv 0(\bmod 3)$. 
If $y \not \equiv 0(\bmod 3), x \equiv u y(\bmod 9)$ and $a x+b y \not \equiv 0(\bmod 3)$, then clearly

$$
\pi^{\prime}=\left\{\begin{array}{c}
-\left(\frac{-3}{y}\right) \omega \pi=\left(\frac{-3}{y}\right)(2 y+(-a x-b y+y) \omega) \\
\text { if } a u+b \equiv 1(\bmod 3), \\
\left(\frac{-3}{y}\right) \omega^{2} \pi=\left(\frac{-3}{y}\right)(-a x-b y+y-(a x+b y+y) \omega) \\
\text { if } a u+b \equiv-1(\bmod 3) .
\end{array}\right.
$$

From this and (5.1) it follows that

$$
\begin{aligned}
& \left.\left(\frac{a x+(b+1) y+2 y \omega}{a_{1} d_{1}^{2} p}\right)_{3}\right)^{2} \omega^{\left(\frac{-3}{y}\right)(-a x-b y+y)(r-s) / 3} \quad \text { if } a u+b \equiv 1(\bmod 3), \\
& = \begin{cases}\left(\frac{\omega}{2^{\alpha+2 \beta} a_{1} d_{1}^{2} p y}\right) \\
\left(\frac{\omega}{2^{\alpha+2 \beta} a_{1} d_{1}^{2} p y}\right)_{3} \omega^{-\left(\frac{-3}{y}\right)(a x+b y+y)(r-s) / 3} \quad \text { if } a u+b \equiv-1(\bmod 3)\end{cases} \\
& =\left(\frac{\omega}{2^{\alpha+2 \beta} a_{1} d_{1}^{2} p y}\right)_{3}^{-\left(\frac{-3}{a u+b}\right)} \omega^{\left(a u+b-\left(\frac{-3}{a u+b}\right)\right)(s-r) / 3} .
\end{aligned}
$$

Observing that

we get

$$
\begin{aligned}
p y & =\left(a\left(\frac{x}{y}\right)^{2}+2 b \frac{x}{y}+c\right)\left(3 \cdot \frac{y-\left(\frac{-3}{y}\right)}{3}+\left(\frac{-3}{y}\right)\right)^{3} \\
& \equiv\left(a u^{2}+2 b u+c\right)\left(\frac{-3}{y}\right)(\bmod 9),
\end{aligned}
$$

$$
\begin{aligned}
& \left(\frac{a x+(b+1) y+2 y \omega}{a_{1} d_{1}^{2} p}\right)_{3} \\
& \quad=\omega^{\left(a u+b-\left(\frac{-3}{a u+b}\right)\right)(s-r) / 3}\left(\frac{\omega}{2^{\alpha+2 \beta} a_{1} d_{1}^{2}\left(a u^{2}+2 b u+c\right)}\right)_{3}^{-\left(\frac{-3}{a u+b}\right)} .
\end{aligned}
$$

Since $a \equiv 0(\bmod 3)$ we must have $d+3 \not \equiv 0(\bmod 3)$ and so $d=b^{2}-a c \equiv 1$ $(\bmod 3)$. Hence,

$$
\left(\frac{\omega}{2^{2 \beta} d_{1}^{2}}\right)_{3}=\left(\frac{\omega}{d+3}\right)_{3}^{2}=\omega^{2(1-d-3) / 3}=\omega^{(d-1) / 3+1}
$$

and

$$
\begin{array}{r}
\left(\frac{\omega}{2^{\alpha+2 \beta} a_{1} d_{1}^{2}\left(a u^{2}+2 b u+c\right)}\right)_{3}=\left(\frac{\omega}{2^{\alpha} a_{1}}\right)_{3}\left(\frac{\omega}{2^{2 \beta} d_{1}^{2}}\right)_{3}\left(\frac{\omega}{a u^{2}+2 b u+c}\right)_{3} \\
=\omega^{\frac{1}{3}\left(1-\left(\frac{-3}{2^{\alpha} a_{1}}\right) 2^{\alpha} a_{1}\right)} \cdot \omega^{\frac{d-1}{3}+1} \cdot \omega^{\frac{1}{3}\left(1-\left(\frac{-3}{2 b u+c}\right)\left(a u^{2}+2 b u+c\right)\right)} .
\end{array}
$$


In view of (5.2),

$$
\left(\frac{a x+(b+1) y+2 y \omega}{a_{1} d_{1}^{2} p}\right)_{3}=\omega^{n_{1}}
$$

where

$$
\begin{aligned}
n_{1}= & -r \frac{a u+b-\left(\frac{-3}{a u+b}\right)}{3}-\left(\frac{-3}{a u+b}\right)\left(\frac{1}{3}\left(1-\left(\frac{-3}{2^{\alpha} a_{1}}\right) 2^{\alpha} a_{1}\right)\right. \\
& \left.+\frac{d-1}{3}+1+\frac{1}{3}\left(1-\left(\frac{-3}{2 b u+c}\right)\left(a u^{2}+2 b u+c\right)\right)\right) \\
= & -\frac{1}{3}\left(r\left(a u+b-\left(\frac{-3}{b}\right)\right)+\left(\frac{-3}{b}\right)\left(d+3-\left(\frac{-3}{2^{\alpha} a_{1}}\right) 2^{\alpha} a_{1}\right)\right. \\
& \left.+\left(\frac{-3}{b}\right)\left(1-\left(\frac{-3}{2 b u+c}\right)\left(a u^{2}+2 b u+c\right)\right)\right) .
\end{aligned}
$$

Since

$$
\begin{aligned}
& r a u-\left(\frac{-3}{b}\right)\left(\frac{-3}{2 b u+c}\right) a u^{2}=a u\left(r-\left(\frac{-3}{b c-u}\right) u\right) \\
& \equiv\left\{\begin{array}{r}
0(\bmod 9) \quad \text { if } a \equiv 0(\bmod 9) \text { or } u \equiv 0(\bmod 3) \\
a(-b c)\left(1-\left(\frac{-3}{2 b c}\right)(-b c)\right) \equiv 0(\bmod 9) \\
\text { if } a \equiv \pm 3(\bmod 9) \text { and } u \equiv-b c(\bmod 3)
\end{array}\right.
\end{aligned}
$$

and

$$
d+3=b^{2}-1-a c+4 \equiv 1-b\left(\frac{-3}{b}\right)-a c+4(\bmod 9),
$$

we see that $n_{1} \equiv f_{1}(u)(\bmod 3)$ and so

$$
\left(\frac{a x+(b+1) y+2 y \omega}{a_{1} d_{1}^{2} p}\right)_{3}=\omega^{f_{1}(u)} .
$$

CASE 3: $a(a x+b y) \not \equiv 0(\bmod 3)$. In this case, $r=0$. Suppose $x \equiv u y$ $(\bmod 9)$. Then

$$
\begin{aligned}
2^{\alpha} a_{1}\left(a u^{2}+2 b u+c\right)= & a^{2} u^{2}+2 a b u+a c \\
& =\left(3 \cdot \frac{a u+b-\left(\frac{-3}{a u+b}\right)}{3}+\left(\frac{-3}{a u+b}\right)\right)^{2}-d \\
& \equiv 1-d-\left((a u+b)\left(\frac{-3}{a u+b}\right)-1\right)(\bmod 9) .
\end{aligned}
$$

From this and (5.2) it follows that 


$$
\begin{aligned}
& \left(\frac{a x+(b+1) y+2 y \omega}{a_{1} d_{1}^{2} p}\right)_{3} \\
& =\left(\frac{\omega}{2^{2 \beta} d_{1}^{2}\left(1-d-\left((a u+b)\left(\frac{-3}{a u+b}\right)-1\right)\right)}\right)_{3}^{-\left(\frac{-3}{a u+b}\right)} \omega^{s\left(a u+b-\left(\frac{-3}{a u+b}\right)\right) / 3}=\omega^{n_{2}},
\end{aligned}
$$

where

$$
\begin{aligned}
n_{2}= & -\frac{1}{3}\left(\frac{-3}{a u+b}\right)\left(1-\left(\frac{-3}{1-d}\right) 2^{2 \beta} d_{1}^{2}\right. \\
& \left.\times\left(1-d-\left((a u+b)\left(\frac{-3}{a u+b}\right)-1\right)\right)\right)+\frac{s\left(a u+b-\left(\frac{-3}{a u+b}\right)\right)}{3} \\
= & \frac{1}{3}\left(\frac{-3}{a u+b}\right)\left(\left((a u+b)\left(\frac{-3}{a u+b}\right)-1\right)\right. \\
& \left.\times\left(s-\left(\frac{-3}{1-d}\right)\right)+\left(2^{\beta} d_{1}\right)^{2}(1-d)\left(\frac{-3}{1-d}\right)-1\right) .
\end{aligned}
$$

Since

$$
\begin{aligned}
& \left(2^{\beta} d_{1}\right)^{2}(1-d)\left(\frac{-3}{1-d}\right)-1 \\
& \quad=\left(\left(2^{\beta} d_{1}\right)^{2}-1\right)\left((1-d)\left(\frac{-3}{1-d}\right)-1\right)+(1-d)\left(\frac{-3}{1-d}\right)+\left(2^{\beta} d_{1}\right)^{2}-2 \\
& \quad \equiv(1-d)\left(\frac{-3}{1-d}\right)-2^{\beta} d_{1}\left(\frac{-3}{2^{\beta} d_{1}}\right)(\bmod 9),
\end{aligned}
$$

we obtain

$$
\left(\frac{a x+(b+1) y+2 y \omega}{a_{1} d_{1}^{2} p}\right)_{3}=\omega^{n_{2}}=\omega^{f_{2}(u)} .
$$

CASE $4: a x+b y \equiv 0(\bmod 9)$. Since $a x+b y \equiv 0(\bmod 3)$ we have $a p=(a x+b y)^{2}-d y^{2} \equiv-d y^{2}(\bmod 9)$. We claim that $a d y \not \equiv 0(\bmod 3)$ and so that $r=s=t=0$.

If $y \equiv 0(\bmod 3)$ then

$$
p=a x^{2}+2 b x y+c y^{2}=(a x+b y) x+(b x+c y) y \equiv 0(\bmod 3) .
$$

Thus, $y \not \equiv 0(\bmod 3)$.

If $a \equiv 0(\bmod 3)$ then $d y^{2} \equiv-a p \equiv 0(\bmod 3)$. Since $y \not \equiv 0(\bmod 3)$ we have $d \equiv 0(\bmod 3)$ and so $d+3 \equiv 0(\bmod 3)$. This contradicts the assumption $(a, d+3)=1$. Hence, $a \not \equiv 0(\bmod 3)$.

By the above, $a y \not \equiv 0(\bmod 3)$ and $d y^{2} \equiv-a p(\bmod 9)$. So $d \not \equiv 0(\bmod 3)$. This proves the assertion.

Now, it is easy to check that

$$
\pi^{\prime}=-\left(\frac{-3}{y}\right) \frac{\pi}{\omega(1-\omega)}=\left(\frac{-3}{y}\right)\left(-y+\frac{a x+b y}{3}+\frac{2(a x+b y)}{3} \omega\right) .
$$


So we have

$$
r=s=t=0, \quad j=k=1 \quad \text { and } \quad B=\left(\frac{-3}{y}\right) \frac{2(a x+b y)}{3} .
$$

This together with (5.1) gives

$$
\left(\frac{a x+(b+1) y+2 y \omega}{a_{1} d_{1}^{2} p}\right)_{3}=\omega^{0}=1 .
$$

CASE $5: a x+b y \equiv \pm 3 y(\bmod 9)$. In this case, $a x+b y \equiv 0(\bmod 3)$. By the above claim we have $r=s=t=0$. It is clear that

$$
\pi^{\prime}=\left\{\begin{array}{c}
-\left(\frac{-3}{y}\right) \frac{\pi}{\omega^{2}(1-\omega)}=\left(\frac{-3}{y}\right)\left(\frac{a x+b y}{3}+y+\left(y-\frac{a x+b y}{3}\right) \omega\right) \\
\text { if } a x+b y \equiv 3 y(\bmod 9), \\
-\left(\frac{-3}{y}\right) \frac{\pi}{1-\omega}=-\left(\frac{-3}{y}\right)\left(\frac{2(a x+b y)}{3}+\left(\frac{a x+b y}{3}+y\right) \omega\right) \\
\text { if } a x+b y \equiv-3 y(\bmod 9) .
\end{array}\right.
$$

Thus, by (5.1),

$$
\begin{aligned}
\left(\frac{a x+(b+1) y+2 y \omega}{a_{1} d_{1}^{2} p}\right)_{3} & \begin{cases}\left(\frac{\omega}{2^{\alpha+2 \beta} a_{1} d_{1}^{2} p y}\right)_{3}^{2-1} & \text { if } a x+b y \equiv 3 y(\bmod 9), \\
\left(\frac{\omega}{2^{\alpha+2 \beta} a_{1} d_{1}^{2} p y}\right)_{3}^{0-1} & \text { if } a x+b y \equiv-3 y(\bmod 9) .\end{cases}
\end{aligned}
$$

Since

$$
\begin{aligned}
2^{\alpha} a_{1}\left(2^{\beta} d_{1}\right)^{2} p y & =a(d+3)^{2}\left(a x^{2}+2 b x y+c y^{2}\right) y \\
& =(d+3)^{2}\left((a x+b y)^{2}-d y^{2}\right) y \equiv\left(d^{2}-3 d\right)(-d) y^{3} \\
& =\left(-d^{3}+3 d^{2}\right) y^{3} \equiv\left(-\left(\frac{-3}{d}\right)+3\right)\left(\frac{-3}{y}\right)(\bmod 9),
\end{aligned}
$$

we obtain

$$
\left(\frac{\omega}{2^{\alpha+2 \beta} a_{1} d_{1}^{2} p y}\right)_{3}=\left(\frac{\omega}{3-\left(\frac{-3}{d}\right)}\right)_{3}=\omega^{\frac{1}{3}\left(1-\left(3-\left(\frac{-3}{d}\right)\right)\left(\frac{-3}{3-\left(\frac{-3}{d}\right)}\right)\right)}=\omega^{\left(\frac{-3}{d}\right)}
$$

and hence

$$
\left(\frac{a x+(b+1) y+2 y \omega}{a_{1} d_{1}^{2} p}\right)_{3}=\omega^{ \pm\left(\frac{-3}{d}\right)} .
$$

This completes the proof.

From Theorem 5.1 we have 
TheOREM 5.2. Let $p$ be a prime greater than $3, d \in\{-1,-2,-5,-6$, $-7,-15\},\left(\frac{d}{p}\right)=1$ and $(s(d))^{2} \equiv d(\bmod p)$. Then $s(d) \in C_{0}(p)$ if and only if $p$ can be represented by one of the corresponding binary quadratic forms in Table 5.1.

Table 5.1

\begin{tabular}{l|r}
\hline$d$ & Binary quadratic forms \\
\hline-1 & $x^{2}+81 y^{2}, 2 x^{2}+2 x y+41 y^{2}$ \\
-2 & $x^{2}+162 y^{2}, 2 x^{2}+81 y^{2}$ \\
-5 & $x^{2}+405 y^{2}, 5 x^{2}+81 y^{2}, 10 x^{2}+10 x y+43 y^{2}, 2 x^{2}+2 x y+203 y^{2}$ \\
-6 & $x^{2}+54 y^{2}, 2 x^{2}+27 y^{2}$ \\
-7 & $x^{2}+567 y^{2}, 7 x^{2}+81 y^{2}, 23 x^{2}+20 x y+29 y^{2}$ \\
-15 & $x^{2}+135 y^{2}, 5 x^{2}+27 y^{2}$ \\
\hline
\end{tabular}

Proof. If $d=-1$ then $\left(\frac{-1}{p}\right)=1$ and so $p=x^{2}+y^{2}$ for some $x, y \in \mathbb{Z}$. Setting $a=1, b=0$ and $c=1$ in Theorem 5.1 we get

$$
\left(\frac{x+y+2 y \omega}{p}\right)_{3}= \begin{cases}\omega^{\left(\frac{-3}{x}\right) \frac{y}{3}} & \text { if } 3 \mid y, \\ \omega^{\left(u-\left(\frac{-3}{u}\right)\right) / 3} & \text { if } 3 \nmid x \text { and } x \equiv u y(\bmod 9), \\ 1 & \text { if } 9 \mid x, \\ \omega^{\mp 1} & \text { if } x \equiv \pm 3 y(\bmod 9) .\end{cases}
$$

Since $y \not \equiv 0(\bmod p)$ and $s(-1) \equiv \pm x / y(\bmod p)$ we see that

$$
\begin{aligned}
s(-1) \in C_{0}(p) & \Leftrightarrow x / y \in C_{0}(p) \\
& \Leftrightarrow\left(\frac{x+y+2 y \omega}{p}\right)_{3}=\left(\frac{x / y+1+2 \omega}{p}\right)_{3}=1 \\
& \Leftrightarrow 9|x, 9| y \text { or } x \equiv\left(\frac{-3}{x y}\right) y(\bmod 9) .
\end{aligned}
$$

Clearly, $p=x^{2}+y^{2}$ with $x, y \in \mathbb{Z}$ and $9 \mid x y$ if and only if $p=x_{1}^{2}+81 y_{1}^{2}$ for some $x_{1}, y_{1} \in \mathbb{Z}$. If $p=x^{2}+y^{2}$ with $x \equiv\left(\frac{-3}{x y}\right) y(\bmod 9)$ then $p=$ $2 x_{1}^{2}+2 x_{1} y_{1}+41 y_{1}^{2}$ for $x_{1}=\frac{1}{9}\left(4 x+5\left(\frac{-3}{x y}\right) y\right)$ and $y_{1}=\frac{1}{9}\left(x-\left(\frac{-3}{x y}\right) y\right)$. Conversely, if $p=2 x_{1}^{2}+2 x_{1} y_{1}+41 y_{1}^{2}$ with $x_{1}, y_{1} \in \mathbb{Z}$ then $p=x^{2}+y^{2}$ for $x=x_{1}+5 y_{1}$ and $y=x_{1}-4 y_{1}$. Also, $x \equiv\left(\frac{-3}{x y}\right) y(\bmod 9)$.

By the above, $s(-1) \in C_{0}(p)$ if and only if $p=x^{2}+81 y^{2}$ or $p=$ $2 x^{2}+2 x y+41 y^{2}$ for some $x, y \in \mathbb{Z}$.

If $d=-2$ then $\left(\frac{-2}{p}\right)=1$ and so $p=x^{2}+2 y^{2}$ for some $x, y \in \mathbb{Z}$. Using the fact that $s(-2) \equiv \pm x / y(\bmod p)$ and Theorem 5.1 we see that

$$
\begin{aligned}
s(-2) \in C_{0}(p) & \Leftrightarrow\left(\frac{x+y+2 y \omega}{p}\right)_{3}=1 \Leftrightarrow 9 \mid x \text { or } 9 \mid y \\
& \Leftrightarrow p=x_{1}^{2}+162 y_{1}^{2} \text { or } p=2 x_{1}^{2}+81 y_{1}^{2}\left(x_{1}, y_{1} \in \mathbb{Z}\right) .
\end{aligned}
$$


If $d=-5$ then $\left(\frac{-5}{p}\right)=1$ and so $p=x^{2}+5 y^{2}$ or $p=3 x^{2}+2 x y+2 y^{2}$ for some $x, y \in \mathbb{Z}$. Using Theorem 5.1 we see that

$$
s(-5) \in C_{0}(p) \Leftrightarrow\left\{\begin{array}{c}
x / y \in C_{0}(p) \Leftrightarrow 9 \mid x \text { or } 9 \mid y \\
\text { if } p=x^{2}+5 y^{2} \\
1+3 x / y \in C_{0}(p) \Leftrightarrow 9 \mid x \text { or } x \equiv-2 y(\bmod 9) \\
\text { if } p=3 x^{2}+2 x y+2 y^{2} .
\end{array}\right.
$$

This yields the result.

If $d=-6$ then $\left(\frac{-6}{p}\right)=1$ and so $p=x^{2}+6 y^{2}$ or $p=2 x^{2}+3 y^{2}$ for some $x, y \in \mathbb{Z}$. Applying Theorem 5.1 we get

$$
s(-6) \in C_{0}(p) \Leftrightarrow \begin{cases}x / y \in C_{0}(p) \Leftrightarrow 3 \mid y & \text { if } p=x^{2}+6 y^{2}, \\ 2 x / y \in C_{0}(p) \Leftrightarrow 3 \mid y & \text { if } p=2 x^{2}+3 y^{2} .\end{cases}
$$

This gives the result.

If $d=-7$ then $\left(\frac{-7}{p}\right)=1$ and so $p=x^{2}+7 y^{2}$ for some $x, y \in \mathbb{Z}$. Applying Theorem 5.1 we see that

$$
s(-7) \in C_{0}(p) \Leftrightarrow x / y \in C_{0}(p) \Leftrightarrow 9|x, 9| y \text { or } x \equiv 4\left(\frac{-3}{x y}\right) y(\bmod 9) .
$$

This yields the desired result.

If $d=-15$ then $\left(\frac{-15}{p}\right)=1$ and hence $p=x^{2}+15 y^{2}$ or $p=5 x^{2}+3 y^{2}$ for some $x, y \in \mathbb{Z}$. In view of Theorem 5.1 we get

$$
s(-15) \in C_{0}(p) \Leftrightarrow \begin{cases}x / y \in C_{0}(p) \Leftrightarrow 3 \mid y & \text { if } p=x^{2}+15 y^{2}, \\ 5 x / y \in C_{0}(p) \Leftrightarrow 3 \mid y & \text { if } p=5 x^{2}+3 y^{2} .\end{cases}
$$

This deduces the result.

Combining the above we prove the theorem.

COROLlaRY 5.1. Let $p$ be a prime greater than 3 .

(i) If $\left(\frac{-1}{p}\right)=1$ then $x^{3}+6 x+4 \equiv 0(\bmod p)$ is solvable if and only if $p=x^{2}+81 y^{2}$ or $p=2 x^{2}+2 x y+41 y^{2}$ for some $x, y \in \mathbb{Z}$.

(ii) If $\left(\frac{-2}{p}\right)=1$ then $x^{3}-9 x-18 \equiv 0(\bmod p)$ is solvable if and only if $p=x^{2}+162 y^{2}$ or $p=2 x^{2}+81 y^{2}$ for some $x, y \in \mathbb{Z}$.

(iii) If $\left(\frac{-6}{p}\right)=1$ then $x^{3}+3 x+2 \equiv 0(\bmod p)$ is solvable if and only if $p=x^{2}+54 y^{2}$ or $p=2 x^{2}+27 y^{2}$ for some $x, y \in \mathbb{Z}$.

(iv) If $\left(\frac{-15}{p}\right)=1$ then $x^{3}+3 x+1 \equiv 0(\bmod p)$ is solvable if and only if $p=x^{2}+135 y^{2}$ or $p=5 x^{2}+27 y^{2}$ for some $x, y \in \mathbb{Z}$.

Proof. If $\left(\frac{-1}{p}\right)=1$, then $(s(-1))^{2} \equiv-1(\bmod p)$ for some $s(-1) \in \mathbb{Z}$. Set $a=-2$ and $b=2$. Then $(6 s(-1))^{2} \equiv-3\left(b^{2}-4 a\right)(\bmod p)$. From Theorems 4.1 and 5.2 we see that 


$$
\begin{aligned}
x^{3}+6 x+4 \equiv & 0(\bmod p) \text { is solvable } \Leftrightarrow 6 s(-1) / 2 \in C_{0}(p) \\
& \left.\Leftrightarrow s(-1) \in C_{0}(p) \text { by Proposition } 2.2(\mathrm{i})\right) \\
& \Leftrightarrow p=x^{2}+81 y^{2} \text { or } p=2 x^{2}+2 x y+41 y^{2}(x, y \in \mathbb{Z}) .
\end{aligned}
$$

This proves (i).

Similarly, by using Theorems 4.1 and 5.2 one can prove (ii)-(iv).

REMARK 5.1. Kronecker $[\mathrm{K}]$ showed that $x^{3}+x+1 \equiv 0(\bmod p)$ is solvable for prime $p$ satisfying $\left(\frac{-31}{p}\right)=1$ if and only if $p=x^{2}+31 y^{2}$ for some integers $x$ and $y$. In 1973, E. Lehmer [L3] proved Corollary 5.1(iv) in the case $p \equiv 1(\bmod 3)$. For recent important papers along this line one may consult $[\mathrm{WH}]$ and $[\mathrm{SW}]$.

Corollary 5.2. Let $p$ be a prime of the form $3 n+1$, and $\varepsilon_{d}$ denote the fundamental unit of the quadratic field $\mathbb{Q}(\sqrt{d})$.

(i) If $d \in\{2,3,5\}$ and $\left(\frac{d}{p}\right)=1$ then $\varepsilon_{d}$ is a cubic residue $(\bmod p)$ if and only if $p=x^{2}+27 d y^{2}$ for some integers $x$ and $y$.

(ii) If $\left(\frac{6}{p}\right)=1$ then $\varepsilon_{6}(=5+2 \sqrt{6})$ is a cubic residue $(\bmod p)$ if and only if $p=x^{2}+162 y^{2}$ for some integers $x$ and $y$.

(iii) If $\left(\frac{15}{p}\right)=1$ then $\varepsilon_{15}(=4+\sqrt{15})$ is a cubic residue $(\bmod p)$ if and only if $p=x^{2}+405 y^{2}$ or $p=10 x^{2}+10 x y+43 y^{2}$ for some integers $x$ and $y$.

(iv) If $\left(\frac{21}{p}\right)=1$ then $\varepsilon_{21}\left(=\frac{1}{2}(5+\sqrt{21})\right)$ is a cubic residue $(\bmod p)$ if and only if $p=x^{2}+567 y^{2}$ or $p=7 x^{2}+81 y^{2}$ for some integers $x$ and $y$.

Proof. Suppose $t^{2} \equiv-3(\bmod p),\left(\frac{-d}{p}\right)=1$ and $(s(-d))^{2} \equiv-d(\bmod p)$. By Theorem 2.2(i), $s(-d) \in C_{0}(p)$ if and only if $(s(-d)-t) /(s(-d)+t)$ is a cubic residue $(\bmod p)$. Observing that

$$
\frac{s(-d)-t}{s(-d)+t}=\frac{(s(-d))^{2}-2 s(-d) t+t^{2}}{(s(-d))^{2}-t^{2}} \equiv \frac{d+3+2 s(-d) t}{d-3}(\bmod p)
$$

and that $(s(-d) t)^{2} \equiv 3 d(\bmod p)$ we find that

$$
s(-d) \in C_{0}(p) \Leftrightarrow \frac{d+3+2 \sqrt{3 d}}{d-3} \text { is a cubic residue }(\bmod p) .
$$

Clearly,

$$
\begin{aligned}
& \varepsilon_{2}=1+\sqrt{2}=\frac{(1+\sqrt{2})^{3}}{3+2 \sqrt{2}}, \quad \varepsilon_{3}=2+\sqrt{3}, \\
& \varepsilon_{5}=\frac{1+\sqrt{5}}{2}=\left(\frac{1+\sqrt{5}}{2}\right)^{3} /\left(\frac{3+\sqrt{5}}{2}\right), \quad \varepsilon_{6}=5+2 \sqrt{6}, \\
& \varepsilon_{15}=4+\sqrt{15}, \quad \varepsilon_{21}=\frac{1}{2}(5+\sqrt{21}) .
\end{aligned}
$$


Hence, combining Theorem 5.2 with (5.3) in the cases $d=6,1,15,2,5,7$ gives the result.

REMARK 5.2. Corollary 5.2(i) was known by E. Lehmer [L3], and the rest of Corollary 5.2 is new. For a general result on the cubic character of quadratic units one may consult [We].

THEOREM 5.3. Let $p$ be a prime of the form $3 n+1,4 p=L^{2}+27 M^{2}$ $(L, M \in \mathbb{Z})$, and $q(d)(q(d)>3)$ a prime divisor of $L^{2}-9 d M^{2}$ or $-d L^{2}+$ $81 M^{2}$

(i) If $k \in \mathbb{Z}$ then $q\left(-3 k^{2}\right)$ is a cubic residue $(\bmod p)$ if and only if $(k-1)\left(k^{2}-1\right)$ is a cubic residue $\left(\bmod q\left(-3 k^{2}\right)\right)$.

(ii) If $d \in\{-1,-2,-5,-6,-7,-15\}$ then $q(d)$ is a cubic residue $(\bmod p)$ if and only if $q(d)$ can be represented by one of the corresponding binary quadratic forms in Table 5.1.

Proof. Suppose $(s(d))^{2} \equiv d(\bmod q(d))$. We first claim that

$$
q(d) \text { is a cubic residue }(\bmod p) \Leftrightarrow s(d) \in C_{0}(q(d)) \text {. }
$$

If $q(d) \mid d$ then $q(d) \mid L M$ and $q(d) \mid s(d)$. From Proposition 2.1 and Corollary 2.1 we see that $s(d) \in C_{0}(q(d))$ and that $q(d)$ is a cubic residue $(\bmod p)$. Since

If $q(d) \nmid d$ then $q(d) \nmid L M$. (Otherwise, $4 p=L^{2}+27 M^{2} \equiv 0\left(\bmod (q(d))^{2}\right)$.)

we have

$$
\left(\frac{L}{3 M}\right)^{2} \equiv d(\bmod q(d)) \quad \text { or } \quad\left(\frac{9 M}{L}\right)^{2} \equiv d(\bmod q(d))
$$

$$
s(d) \equiv \pm \frac{L}{3 M} \quad \text { or } \quad s(d) \equiv \pm \frac{9 M}{L}(\bmod q(d)) .
$$

Now, applying Corollary 2.1 and Proposition 2.2(i) we see that

$$
q(d) \text { is a cubic residue }(\bmod p) \Leftrightarrow \frac{L}{3 M} \in C_{0}(q(d)) \Leftrightarrow s(d) \in C_{0}(q(d)) .
$$

This proves the assertion.

Now let us consider (i). Suppose $d=-3 k^{2}$ for some $k \in \mathbb{Z}$. If $k \equiv \pm 1$ $(\bmod q(d))$ then $d \equiv-3(\bmod q(d))$ and so $4 p=L^{2}+27 M^{2} \equiv 0(\bmod q(d))$. This implies $q(d)=p$. So $q(d)$ is a cubic residue $(\bmod p)$. If $k \not \equiv \pm 1$ $(\bmod q(d))$, by $(5.3)$ and $(5.4)$ we see that

$q(d)$ is a cubic residue $(\bmod p) \Leftrightarrow s(d) \in C_{0}(q(d))$

$$
\begin{aligned}
& \Leftrightarrow \frac{3 k^{2}+3+2 \cdot 3 k}{3 k^{2}-3}\left(=\frac{k+1}{k-1}\right) \text { is a cubic residue }(\bmod q(d)) \\
& \Leftrightarrow(k-1)\left(k^{2}-1\right) \text { is a cubic residue }(\bmod q(d)) .
\end{aligned}
$$

This proves (i).

(ii) follows from (5.4) and Theorem 5.2. 
Remark 5.3. When $q(d)$ is a prime divisor of $L^{2}+9 d M^{2}$ Federighi and Roll [FR] conjectured Theorem 5.3(ii) in the cases $d=6,15$. Ph. Barkan $[\mathrm{Ba}]$ showed how to prove their conjecture about primes $q(d) \equiv 1(\bmod 3)$.

In 1992, using class field theory Spearman and Williams [SW] proved the following important result:

(5.5) Suppose $p>3$ is a prime and $x^{3}+A x^{2}+B x+C(A, B, C \in \mathbb{Z})$ is irreducible over the rational field $\mathbb{Q}$. If the discriminant $D=A^{2} B^{2}-4 B^{3}-$ $4 A^{3} C-27 C^{2}+18 A B C$ is not a perfect square such that $\left(\frac{D}{p}\right)=1$, and $H(D)$ is the form class group of classes of primitive, integral binary quadratic forms of discriminant $D$, then the cubic congruence $x^{3}+A x^{2}+B x+C \equiv 0$ $(\bmod p)$ is solvable if and only if $p$ can be represented by one of the third (composition) powers of forms in $H(D)$.

From (5.5) we have

Lemma 5.1. Assume that $p>3$ is a prime, $m, n \in \mathbb{Z}, 2 \mid m n, p \nmid m n$, $m^{3} n \neq-2,4,64,108,250, m^{3} n / 2-27 \notin\left\{k^{2} \mid k \in \mathbb{Z}\right\}$ and $\left(\frac{m^{3} n / 2-27}{p}\right)=1$. Then the cubic congruence $x^{3}-\frac{m n}{2} x-n \equiv 0(\bmod p)$ is solvable if and only if $p$ can be represented by one of the third powers of primitive integral binary quadratic forms of discriminant $\left(m^{3} n / 2-27\right) n^{2}$.

P r o of. Clearly the discriminant of $x^{3}-\frac{m n}{2} x-n$ is given by

$$
D=-4\left(-\frac{m n}{2}\right)^{3}-27(-n)^{2}=\left(\frac{m^{3} n}{2}-27\right) n^{2} .
$$

Since $D$ is not a square, by (5.5) it is sufficient to prove that $x^{3}-\frac{m n}{2} x-n \neq 0$ for any integer $x$.

If $t \in \mathbb{Z}$ and $t^{3}-\frac{m n}{2} t-n=0$, then $n=s t$ for some $s \in \mathbb{Z}$. Since $n \not \equiv 0$ $(\bmod p)$ we have $s t \neq 0$ and so $t^{2}-\frac{m s t}{2}-s=0$. This implies $t \mid 2 s$. Write $2 s=r t$. Then $t^{2}-\frac{m r}{4} t^{2}-\frac{r t}{2}=0$. Namely, $4 t-m r t-2 r=0$. It then follows that $4 t=k r$ for some $k \in \mathbb{Z}$. Observing that $r=2 s / t \neq 0$ we find $k(4-m r)=8$ and hence $k \in\{ \pm 1, \pm 2, \pm 4, \pm 8\}$. Since

$$
m^{3} n=m^{3} \cdot \frac{r}{2} \cdot\left(\frac{k r}{4}\right)^{2}=\frac{(m r)^{3}}{32} k^{2}=\frac{k^{2}}{32}\left(4-\frac{8}{k}\right)^{3}=\frac{2(k-2)^{3}}{k}
$$

and $k \in\{ \pm 1, \pm 2, \pm 4, \pm 8\}$ we get $m^{3} n \in\{-2,54,0,64,4,108,250\}$. This contradicts the assumption. Thus, $x^{3}-\frac{m n}{2} x-n \neq 0$ for any $x \in \mathbb{Z}$. This completes the proof.

Now we can give

TheOREM 5.4. If $p>3$ is a prime, $d \in \mathbb{Z}, d \neq 3, d \not \equiv-3(\bmod p), d \notin$ $\left\{k^{2} \mid k \in \mathbb{Z}\right\},\left(\frac{d}{p}\right)=1,(s(d))^{2} \equiv d(\bmod p)$ and $18(d+3)=m^{3} n$ with $m, n \in \mathbb{Z}$, then $s(d) \in C_{0}(p)$ if and only if $p$ can be represented by one of 
the third powers of primitive integral binary quadratic forms of discriminant $9 d n^{2}$.

Proof. It follows from Lemma 4.1 that

$$
\begin{aligned}
s(d) \in & C_{0}(p) \\
& \Leftrightarrow x^{3}-9\left((s(d))^{2}+3\right) x-18\left((s(d))^{2}+3\right) \equiv 0(\bmod p) \text { is solvable } \\
& \Leftrightarrow x^{3}-9(d+3) x-18(d+3) \equiv 0(\bmod p) \text { is solvable } \\
& \Leftrightarrow(m y)^{3}-\frac{m^{3} n}{2} m y-m^{3} n \equiv 0(\bmod p) \text { is solvable } \\
& \Leftrightarrow y^{3}-\frac{m n}{2} y-n \equiv 0(\bmod p) \text { is solvable. }
\end{aligned}
$$

Since $\left(m^{3} n / 2-27\right) n^{2}=9 d n^{2}$ and $m^{3} n=18(d+3) \neq-2,4,64,108,250$, applying Lemma 5.1 we obtain the result.

Corollary 5.3. If $p$ is a prime, $p \equiv 1(\bmod 3), k \in \mathbb{Z}, k \neq 0, \pm 1$ $(\bmod p)$ and $2\left(k^{2}-1\right)=m^{3} n(m, n \in \mathbb{Z})$, then $\frac{k+1}{k-1}$ is a cubic residue $(\bmod$ $p$ ) if and only if $p$ can be represented by one of the third powers of primitive integral binary quadratic forms of discriminant $-27 k^{2} n^{2}$.

Proof. Suppose $d=-3 k^{2}$ and $t^{2} \equiv-3(\bmod p)$. Clearly, $\left(\frac{d}{p}\right)=1$, $d \not \equiv-3(\bmod p)$ and $(-k t)^{2} \equiv d(\bmod p)$. By Theorem $2.2(\mathrm{i}),-k t \in C_{0}(p)$ if and only if $\frac{k+1}{k-1}\left(=\frac{-k t-t}{-k t+t}\right)$ is a cubic residue $(\bmod p)$. Also, $18(d+3)=$ $18\left(3-3 k^{2}\right)=(-3 m)^{3} n$ and $9 d n^{2}=-27 k^{2} n^{2}$. So the result follows from Theorem 5.4.

Corollary 5.4. Let $p$ be a prime of the form $3 n+1,4 p=L^{2}+27 M^{2}$ $(L, M \in \mathbb{Z}), d \in \mathbb{Z}, d \notin\left\{k^{2} \mid k \in \mathbb{Z}\right\}, d \neq 3, d \not \equiv-3(\bmod p)$ and $18(d+3)=$ $m^{3} n(m, n \in \mathbb{Z})$. If $q(d)$ is a prime divisor of $L^{2}-9 d M^{2}$ or $-d L^{2}+81 M^{2}$ satisfying $q(d) \neq 2,3$ and $q(d) \nmid d$, then $q(d)$ is a cubic residue $(\bmod p)$ if and only if $q(d)$ can be represented by one of the third powers of primitive integral binary quadratic forms of discriminant $9 \mathrm{dn}^{2}$.

Proof. This is immediate from (5.4) and Theorem 5.4.

6. Applications to Lucas series. Let $a$ and $b$ be two real numbers. The Lucas sequences $\left\{u_{n}(a, b)\right\}$ and $\left\{v_{n}(a, b)\right\}$ are defined as follows:

$$
\begin{aligned}
u_{0}(a, b) & =0, \quad u_{1}(a, b)=1, \\
u_{n+1}(a, b) & =b u_{n}(a, b)-a u_{n-1}(a, b) \quad(n \geq 1) ; \\
v_{0}(a, b) & =2, \quad v_{1}(a, b)=b, \\
v_{n+1}(a, b) & =b v_{n}(a, b)-a v_{n-1}(a, b) \quad(n \geq 1) .
\end{aligned}
$$


It is well known that

$$
\text { (6.3) } u_{n}(a, b)=\frac{1}{\sqrt{b^{2}-4 a}}\left(\left(\frac{b+\sqrt{b^{2}-4 a}}{2}\right)^{n}-\left(\frac{b-\sqrt{b^{2}-4 a}}{2}\right)^{n}\right)
$$

and that

$$
v_{n}(a, b)=\left(\frac{b+\sqrt{b^{2}-4 a}}{2}\right)^{n}+\left(\frac{b-\sqrt{b^{2}-4 a}}{2}\right)^{n} .
$$

Suppose that $p$ is a prime greater than 3 . It is the purpose of this section to determine $u_{\left(p-\left(\frac{-3}{p}\right)\right) / 3}(a, b)(\bmod p)$ and $v_{\left(p-\left(\frac{-3}{p}\right)\right) / 3}(a, b)(\bmod p)$.

Theorem 6.1. Let $p>3$ be a prime, $a, b \in \mathbb{Z}_{p}, p \nmid a b,\left(\frac{-3\left(b^{2}-4 a\right)}{p}\right)=1$ and $s^{2} \equiv-3\left(b^{2}-4 a\right)(\bmod p)$. Then

$$
u_{\left(p-\left(\frac{-3}{p}\right)\right) / 3}(a, b) \equiv \begin{cases}0(\bmod p) & \text { if } s / b \in C_{0}(p), \\ \pm \frac{3}{s}(-a)^{-[p / 3]}(\bmod p) & \text { if } \pm s / b \in C_{1}(p)\end{cases}
$$

and

$$
v_{\left(p-\left(\frac{-3}{p}\right)\right) / 3}(a, b) \equiv \begin{cases}2 a^{-[p / 3]}(\bmod p) & \text { if } s / b \in C_{0}(p), \\ -a^{-[p / 3]}(\bmod p) & \text { if } s / b \notin C_{0}(p) .\end{cases}
$$

Proof. Set $k=-3 b / s$. For $n \in \mathbb{Z}^{+}$it is clear that

$$
\begin{aligned}
u_{n}(a, b) & =\frac{1}{\sqrt{b^{2}-4 a}}\left(\left(\frac{b+\sqrt{b^{2}-4 a}}{2}\right)^{n}-\left(\frac{b-\sqrt{b^{2}-4 a}}{2}\right)^{n}\right) \\
& =\frac{2}{2^{n} \sqrt{b^{2}-4 a}} \sum_{r=0}^{[(n-1) / 2]}\left(\begin{array}{c}
n \\
2 r+1
\end{array}\right) b^{n-2 r-1}\left(\sqrt{b^{2}-4 a}\right)^{2 r+1} \\
& =\frac{2}{2^{n}} \sum_{r=0}^{[(n-1) / 2]}\left(\begin{array}{c}
n \\
2 r+1
\end{array}\right) b^{n-2 r-1}\left(b^{2}-4 a\right)^{r} \\
& \equiv \frac{2}{2^{n}} \sum_{r=0}^{[(n-1) / 2]}\left(\begin{array}{c}
n \\
2 r+1
\end{array}\right) b^{n-2 r-1}\left(\frac{s(1+2 \omega)}{-3}\right)^{2 r+1} \frac{-3}{s(1+2 \omega)} \\
& =\frac{-3}{s(1+2 \omega)}\left(\left(\frac{b+s(1+2 \omega) /(-3)}{2}\right)^{n}-\left(\frac{b-s(1+2 \omega) /(-3)}{2}\right)^{n}\right) \\
& =\frac{\omega(1-\omega)}{s}\left(-\frac{s}{6}\right)^{n}\left((k+1+2 \omega)^{n}-(k-1-2 \omega)^{n}\right)(\bmod p) .
\end{aligned}
$$

Similarly,

$$
\begin{aligned}
v_{n}(a, b) & =\frac{2}{2^{n}} \sum_{r=0}^{[n / 2]}\left(\begin{array}{c}
n \\
2 r
\end{array}\right) b^{n-2 r}\left(b^{2}-4 a\right)^{r} \\
& \equiv \frac{2}{2^{n}} \sum_{r=0}^{[n / 2]}\left(\begin{array}{c}
n \\
2 r
\end{array}\right) b^{n-2 r}\left(\frac{s(1+2 \omega)}{-3}\right)^{2 r}
\end{aligned}
$$




$$
\begin{aligned}
& =\left(\frac{b+s(1+2 \omega) /(-3)}{2}\right)^{n}+\left(\frac{b-s(1+2 \omega) /(-3)}{2}\right)^{n} \\
& =\left(-\frac{s}{6}\right)^{n}\left((k+1+2 \omega)^{n}+(k-1-2 \omega)^{n}\right)(\bmod p) .
\end{aligned}
$$

If $p \equiv 1(\bmod 3)$, we may write $p=\lambda \bar{\lambda}$ with $\lambda \in \mathbb{Z}[\omega]$ and $\lambda \equiv 2(\bmod 3)$. By Lemma 2.2(i),

$$
\begin{aligned}
\left(k^{2}+3\right)^{(p-1) / 3} & \left((k-1-2 \omega)^{(p-1) / 3} \pm(k+1+2 \omega)^{(p-1) / 3}\right) \\
& \equiv\left(\frac{\left(k^{2}+3\right)(k-1-2 \omega)}{\lambda}\right)_{3} \pm\left(\frac{\left(k^{2}+3\right)(k+1+2 \omega)}{\lambda}\right)_{3} \\
& =\left(\frac{k+1+2 \omega}{p}\right)_{3} \pm\left(\frac{k+1+2 \omega}{p}\right)_{3}^{-1}(\bmod \lambda) .
\end{aligned}
$$

Hence, by the above and Fermat's little theorem we get

$$
\begin{aligned}
u_{(p-1) / 3}(a, b) \equiv & -\frac{\omega(1-\omega)}{s}\left(-\frac{s}{6}\right)^{(p-1) / 3}\left(k^{2}+3\right)^{-(p-1) / 3} \\
& \times\left(\left(\frac{k+1+2 \omega}{p}\right)_{3}-\left(\frac{k+1+2 \omega}{p}\right)_{3}^{-1}\right) \\
\equiv & \begin{cases}0(\bmod \lambda) & \text { if } k \in C_{0}(p), \\
\pm \frac{3}{s}(-a)^{(p-1) / 3}(\bmod \lambda) & \text { if } \pm k \in C_{1}(p)\end{cases}
\end{aligned}
$$

and

$$
\begin{aligned}
& v_{(p-1) / 3}(a, b) \\
& \equiv\left(-\frac{s}{6}\right)^{(p-1) / 3}\left(k^{2}+3\right)^{-(p-1) / 3}\left(\left(\frac{k+1+2 \omega}{p}\right)_{3}+\left(\frac{k+1+2 \omega}{p}\right)_{3}^{-1}\right) \\
& \equiv \begin{cases}2 a^{-(p-1) / 3}(\bmod \lambda) & \text { if } k \in C_{0}(p), \\
-a^{-(p-1) / 3}(\bmod \lambda) & \text { if } k \in C_{1}(p) \cup C_{2}(p) .\end{cases}
\end{aligned}
$$

Since both sides of the above congruences are rational, the congruences are also true when $\lambda$ is replaced by $p(=N \lambda)$.

If $p \equiv 2(\bmod 3)$, it follows from Lemma $2.2($ ii) that $(k+1+2 \omega)^{(p+1) / 3} \pm(k-1-2 \omega)^{(p+1) / 3}$

$$
\equiv\left(k^{2}+3\right)^{-(p-2) / 3}\left(\left(\frac{k+1+2 \omega}{p}\right)_{3} \pm\left(\frac{k+1+2 \omega}{p}\right)_{3}^{-1}\right)(\bmod p) .
$$

From this and the above it follows that

$$
\begin{aligned}
u_{(p+1) / 3}(a, b) \equiv & \frac{\omega(1-\omega)}{s}\left(-\frac{s}{6}\right)^{(p+1) / 3}\left(k^{2}+3\right)^{-(p-2) / 3} \\
& \times\left(\left(\frac{k+1+2 \omega}{p}\right)_{3}-\left(\frac{k+1+2 \omega}{p}\right)_{3}^{-1}\right)
\end{aligned}
$$




$$
\equiv \begin{cases}0(\bmod p) & \text { if } k \in C_{0}(p), \\ \pm \frac{3}{s}(-a)^{-(p-2) / 3}(\bmod p) & \text { if } \pm k \in C_{1}(p)\end{cases}
$$

and

$$
\begin{aligned}
v_{(p+1) / 3}(a, b) & \\
& \equiv\left(-\frac{s}{6}\right)^{(p+1) / 3}\left(k^{2}+3\right)^{-(p-2) / 3}\left(\left(\frac{k+1+2 \omega}{p}\right)_{3}+\left(\frac{k+1+2 \omega}{p}\right)_{3}^{-1}\right) \\
& \equiv \begin{cases}2 a^{-(p-2) / 3}(\bmod p) & \text { if } k \in C_{0}(p), \\
-a^{-(p-2) / 3}(\bmod p) & \text { if } k \in C_{1}(p) \cup C_{2}(p) .\end{cases}
\end{aligned}
$$

To complete the proof, we note that

$$
k^{2}+3 \equiv \frac{36 a}{-3\left(b^{2}-4 a\right)} \not \equiv 0(\bmod p)
$$

and $k \in C_{i}(p)$ if and only if $s / b \in C_{i}(p)$ by Proposition 2.2(i).

Corollary 6.1. Let $p>3$ be a prime, $k \in \mathbb{Z}_{p}$ and $k\left(k^{2}+3\right) \not \equiv 0$ $(\bmod p)$. Then

$$
u_{\left(p-\left(\frac{-3}{p}\right)\right) / 3}\left(3 k^{2}+9,6\right) \equiv \begin{cases}0(\bmod p) & \text { if } k \in C_{0}(p), \\ \frac{1}{2 k}\left(-3 k^{2}-9\right)^{-[p / 3]}(\bmod p) & \text { if } k \in C_{1}(p), \\ -\frac{1}{2 k}\left(-3 k^{2}-9\right)^{-[p / 3]}(\bmod p) & \text { if } k \in C_{2}(p)\end{cases}
$$

and

$$
v_{\left(p-\left(\frac{-3}{p}\right)\right) / 3}\left(3 k^{2}+9,6\right) \equiv \begin{cases}2\left(3 k^{2}+9\right)^{-[p / 3]}(\bmod p) & \text { if } k \in C_{0}(p), \\ -\left(3 k^{2}+9\right)^{-[p / 3]}(\bmod p) & \text { if } k \notin C_{0}(p) .\end{cases}
$$

Corollary 6.2. Let $p>3$ be a prime, $d \in \mathbb{Z}_{p}, d \not \equiv-3(\bmod p),\left(\frac{d}{p}\right)=1$ and $(s(d))^{2} \equiv d(\bmod p)$. Then $s(d) \in C_{0}(p)$ if and only if $u_{\left(p-\left(\frac{-3}{p}\right)\right) / 3}(3 d+$ $9,6) \equiv 0(\bmod p)$.

Proof. Set $k=s(d)$. Then $u_{n}\left(3 k^{2}+9,6\right) \equiv u_{n}(3 d+9,6)(\bmod p)$ by (6.1). Hence the result follows from Corollary 6.1.

Corollary 6.3. Let $p>3$ be a prime, $a, b \in \mathbb{Z}_{p}, p \nmid a b$ and $\left(\frac{-3\left(b^{2}-4 a\right)}{p}\right)$ $=1$. Then the congruence $x^{3}-3 a x-a b \equiv 0(\bmod p)$ is solvable if and only if $u_{\left(p-\left(\frac{-3}{p}\right)\right) / 3}(a, b) \equiv 0(\bmod p)$.

Pr o of. Suppose $s^{2} \equiv-3\left(b^{2}-4 a\right)(\bmod p)$. It then follows from Theorem 6.1 that $s / b \in C_{0}(p)$ if and only if $u_{\left(p-\left(\frac{-3}{p}\right)\right) / 3}(a, b) \equiv 0(\bmod p)$. This together with Theorem 4.1 gives the result.

Corollary 6.4. Let $p>3$ be a prime, $a, b \in \mathbb{Z}, p \nmid a b$ and $\left(\frac{-3\left(b^{2}-4 a\right)}{p}\right)$ $=1$. If $-3\left(b^{2}-4 a\right)$ is not a square and $x^{3}-3 a x-a b$ is irreducible over 
$\mathbb{Q}$, then $p \mid u_{\left(p-\left(\frac{-3}{p}\right)\right) / 3}(a, b)$ if and only if $p$ can be represented by one of the third powers of primitive integral binary quadratic forms of discriminant $-27 a^{2}\left(b^{2}-4 a\right)$.

Proof. Since the discriminant of $x^{3}-3 a x-a b$ is $-27 a^{2}\left(b^{2}-4 a\right)$ the result follows from (5.5) and Corollary 6.3.

Let $\left\{F_{n}\right\}$ and $\left\{L_{n}\right\}$ be defined by

$$
F_{0}=0, \quad F_{1}=1, \quad F_{n+1}=F_{n}+F_{n-1} \quad(n \geq 1)
$$

and

$$
L_{0}=2, \quad L_{1}=1, \quad L_{n+1}=L_{n}+L_{n-1} \quad(n \geq 1) .
$$

It is well known that $\left\{F_{n}\right\}$ is the Fibonacci sequence and that $\left\{L_{n}\right\}$ is the Lucas sequence.

From Theorems 5.1 and 6.1 we have

THEOREM 6.2. Let $p>5$ be a prime for which $\left(\frac{-15}{p}\right)=1$ and hence $p=x^{2}+15 y^{2}$ or $p=5 x^{2}+3 y^{2}$ for some $x, y \in \mathbb{Z}$ according as $p \equiv 1(\bmod 3)$ or $p \equiv 2(\bmod 3)$. Then

$$
F_{\left(p-\left(\frac{-3}{p}\right)\right) / 3} \equiv \begin{cases}0(\bmod p) & \text { if } y \equiv 0(\bmod 3), \\ -\frac{x}{\left(2+3\left(\frac{-3}{p}\right)\right) y}(\bmod p) & \text { if } y \equiv x(\bmod 3)\end{cases}
$$

and

$$
L_{\left(p-\left(\frac{-3}{p}\right)\right) / 3} \equiv \begin{cases}2\left(\frac{-3}{p}\right)(\bmod p) & \text { if } y \equiv 0(\bmod 3) \\ -\left(\frac{-3}{p}\right)(\bmod p) & \text { if } y \neq 0(\bmod 3)\end{cases}
$$

Proof. Suppose $s=\left(3-2\left(\frac{-3}{p}\right)\right) \frac{x}{y}$. Then $s^{2} \equiv-15(\bmod p)$. Since $F_{n}=u_{n}(-1,1)$ and $L_{n}=v_{n}(-1,1)$, it follows from Theorem 6.1 that

$$
F_{\left(p-\left(\frac{-3}{p}\right)\right) / 3} \equiv \begin{cases}0(\bmod p) & \text { if } s \in C_{0}(p) \\ \pm \frac{3}{s}(\bmod p) & \text { if } \pm s \in C_{1}(p)\end{cases}
$$

and that

$$
L_{\left(p-\left(\frac{-3}{p}\right)\right) / 3} \equiv \begin{cases}2(-1)^{-[p / 3]}=2\left(\frac{-3}{p}\right)(\bmod p) & \text { if } s \in C_{0}(p), \\ -(-1)^{-[p / 3]}=\left(\frac{-3}{p}\right)(\bmod p) & \text { if } s \notin C_{0}(p) .\end{cases}
$$


From Theorem 5.1 we know that

$$
\left(\frac{s+1+2 \omega}{p}\right)_{3}=\left(\frac{s y+y+2 y \omega}{p}\right)_{3}=\left\{\begin{aligned}
1 & \text { if } y \equiv 0(\bmod 3) \\
\omega & \text { if } x \equiv\left(\frac{-3}{p}\right) y(\bmod 3), \\
\omega^{2} & \text { if } x \equiv-\left(\frac{-3}{p}\right) y(\bmod 3) .
\end{aligned}\right.
$$

Hence, $s \in C_{0}(p)$ if and only if $y \equiv 0(\bmod 3)$.

If $y \not \equiv 0(\bmod 3)$ then $x \equiv \pm\left(\frac{-3}{p}\right) y(\bmod 3)$ and so $\pm s \in C_{1}(p)$ by the above. Since

$$
\frac{3}{s}=\frac{3 y}{\left(3-2\left(\frac{-3}{p}\right)\right) x} \equiv-\frac{x}{\left(3+2\left(\frac{-3}{p}\right)\right) y}=-\left(\frac{-3}{p}\right) \frac{x}{\left(3\left(\frac{-3}{p}\right)+2\right) y}(\bmod p)
$$

we obtain

$$
F_{\left(p-\left(\frac{-3}{p}\right)\right) / 3} \equiv \begin{cases}-\frac{x}{5 y}(\bmod p) & \text { if } p \equiv 1(\bmod 3) \text { and } x \equiv y(\bmod p) \\ \frac{x}{y}(\bmod p) & \text { if } p \equiv 2(\bmod 3) \text { and } x \equiv y(\bmod p)\end{cases}
$$

This completes the proof.

LEMMA 6.1. Let $p$ be a prime greater than $3, a, b \in \mathbb{Z}, u_{n}=u_{n}(a, b), v_{n}=$ $v_{n}(a, b)$ and $a b\left(b^{2}-4 a\right) \not \equiv 0(\bmod p)$. Then

(a) $p \mid u_{p-\left(\frac{-3}{p}\right)}$ if and only if $\left(\frac{-3\left(b^{2}-4 a\right)}{p}\right)=1$.

(b) $p \mid u_{n}$ if and only if $v_{2 n} \equiv 2 a^{n}(\bmod p)$.

Proof. From $[\mathrm{D}]$ and $[\mathrm{R}]$ we know that

$$
u_{p-\left(\frac{b^{2}-4 a}{p}\right)} \equiv 0(\bmod p), \quad u_{p} \equiv\left(\frac{b^{2}-4 a}{p}\right)(\bmod p) .
$$

Thus,

$$
\begin{aligned}
u_{p+\left(\frac{b^{2}-4 a}{p}\right)} & = \begin{cases}b u_{p}-a u_{p-1} \equiv b(\bmod p) & \text { if }\left(\frac{b^{2}-4 a}{p}\right)=1, \\
\frac{1}{a}\left(b u_{p}-u_{p+1}\right) \equiv-\frac{b}{a}(\bmod p) & \text { if }\left(\frac{b^{2}-4 a}{p}\right)=-1\end{cases} \\
& \neq 0(\bmod p) .
\end{aligned}
$$

It then follows that

$$
p \mid u_{p-\left(\frac{-3}{p}\right)} \Leftrightarrow\left(\frac{-3}{p}\right)=\left(\frac{b^{2}-4 a}{p}\right) \Leftrightarrow\left(\frac{-3\left(b^{2}-4 a\right)}{p}\right)=1 .
$$

This proves part (a).

Now consider part (b). According to $[\mathrm{D}]$ and $[\mathrm{R}]$ we have

$$
u_{2 n}=u_{n} v_{n}, \quad v_{2 n}=v_{n}^{2}-2 a^{n} \text {, }
$$




$$
v_{n}^{2}-\left(b^{2}-4 a\right) u_{n}^{2}=4 a^{n} .
$$

Thus,

$$
p \mid u_{n} \Leftrightarrow v_{n}^{2} \equiv 4 a^{n}(\bmod p) \Leftrightarrow v_{2 n} \equiv 2 a^{n}(\bmod p) .
$$

This concludes the proof.

Using Lemma 6.1 and Theorem 6.2 we have

Corollary 6.5. Let $p$ be a prime greater than 5. Then

(i) $p \mid F_{\left(p-\left(\frac{-3}{p}\right)\right) / 3}$ if and only if $p$ can be represented by $x^{2}+135 y^{2}$ or $5 x^{2}+27 y^{2}$ according as $p \equiv 1(\bmod 3)$ or $p \equiv 2(\bmod 3)$.

(ii) $p \mid F_{\left(p-\left(\frac{-3}{p}\right)\right) / 6}$ if and only if $p$ can be represented by $x^{2}+540 y^{2}$ or $5 x^{2}+108 y^{2}$ according as $p \equiv 1(\bmod 3)$ or $p \equiv 2(\bmod 3)$.

Pro of. It is well known that (see [D], [SS], [R]) $F_{n} \mid F_{m n}$ for $m, n \in \mathbb{Z}^{+}$. Thus $F_{\left(p-\left(\frac{-3}{p}\right)\right) / 3} \mid F_{p-\left(\frac{-3}{p}\right)}$. If $p \mid F_{\left(p-\left(\frac{-3}{p}\right)\right) / 3}$ then $p \mid F_{p-\left(\frac{-3}{p}\right)}$. Applying Lemma 6.1 we find $\left(\frac{-15}{p}\right)=1$ and so $p=A^{2}+15 B^{2}$ or $p=5 A^{2}+3 B^{2}$ for some $A, B \in \mathbb{Z}$. It then follows from Theorem 6.2 that $3 \mid B$. Hence $p=x^{2}+135 y^{2}$ or $p=5 x^{2}+27 y^{2}$ for some $x, y \in \mathbb{Z}$.

Conversely, if $p$ is represented by $x^{2}+135 y^{2}$ or $5 x^{2}+27 y^{2}$, then $\left(\frac{-15}{p}\right)$ $=1$. Applying Theorem 6.2 we find $p \mid F_{\left(p-\left(\frac{-3}{p}\right)\right) / 3}$. This proves (i).

Let us consider (ii). If $p \mid F_{\left(p-\left(\frac{-3}{p}\right)\right) / 6}$ then $p \mid F_{p-\left(\frac{-3}{p}\right)}$ and so $\left(\frac{-15}{p}\right)=1$ by Lemma 6.1. If $p$ is represented by $x^{2}+540 y^{2}$ or $5 x^{2}+108 y^{2}$, we also have $\left(\frac{-15}{p}\right)=1$. Hence, we may assume $\left(\frac{-15}{p}\right)=1$ and so $p=x^{2}+15 y^{2}$ or $p=5 x^{2}+3 y^{2}$ for some $x, y \in \mathbb{Z}$. It then follows from Lemma 6.1 and Theorem 6.2 that

$$
\begin{aligned}
p \mid F_{\left(p-\left(\frac{-3}{p}\right)\right) / 6} & \Leftrightarrow L_{\left.\left(p-\frac{-3}{p}\right)\right) / 3} \equiv 2(-1)^{\left(p-\left(\frac{-3}{p}\right)\right) / 6}(\bmod p) \\
\Leftrightarrow(-1)^{\left(p-\left(\frac{-3}{p}\right)\right) / 6}=\left(\frac{-3}{p}\right) \text { and } 3 \mid y & \\
& \Leftrightarrow p=\left\{\begin{array}{l}
A^{2}+135 B^{2} \equiv 1(\bmod 12)(A, B \in \mathbb{Z}) \text { if } p \equiv 1(\bmod 3), \\
5 A^{2}+27 B^{2} \equiv 5(\bmod 12)(A, B \in \mathbb{Z}) \text { if } p \equiv 2(\bmod 3)
\end{array}\right. \\
& \Leftrightarrow p=A^{2}+135 B^{2} \text { or } p=5 A^{2}+27 B^{2} \text { with } B \equiv 0(\bmod 2) \\
& \Leftrightarrow p=x^{2}+540 y^{2} \text { or } p=5 x^{2}+108 y^{2} \text { for some } x, y \in \mathbb{Z} .
\end{aligned}
$$

This completes the proof.

REMARK 6.1. In [L3], [L4] E. Lehmer proved Corollary 6.5(i) in the case $p \equiv 1(\bmod 12)$. For the criteria for $p \mid F_{(p-1) / 4}($ if $p \equiv 1(\bmod 4)$ is a prime $)$ one may consult [L6], [SS]. 
Now we point out similar results for the Pell sequence. The Pell sequence $\left\{P_{n}\right\}$ and its companion $\left\{Q_{n}\right\}$ are given by

$$
P_{0}=0, \quad P_{1}=1, \quad P_{n+1}=2 P_{n}+P_{n-1} \quad(n \geq 1)
$$

and

$$
Q_{0}=2, \quad Q_{1}=2, \quad Q_{n+1}=2 Q_{n}+Q_{n-1} \quad(n \geq 1) .
$$

Clearly, $P_{n}=u_{n}(-1,2)$ and $Q_{n}=v_{n}(-1,2)$.

Using Theorems 6.1 and 5.1 one can similarly prove

TheOREM 6.3. Let $p>3$ be a prime such that $\left(\frac{-6}{p}\right)=1$ and hence $p=x^{2}+6 y^{2}$ or $p=2 x^{2}+3 y^{2}$ for some $x, y \in \mathbb{Z}$ according as $p \equiv 1(\bmod 3)$ or $p \equiv 2(\bmod 3)$. Then

$$
P_{\left(p-\left(\frac{-3}{p}\right)\right) / 3} \equiv \begin{cases}0(\bmod p) & \text { if } y \equiv 0(\bmod 3), \\ \frac{x}{\left(1+3\left(\frac{-3}{p}\right)\right) y}(\bmod p) & \text { if } y \equiv x(\bmod 3)\end{cases}
$$

and

$$
Q_{\left.\left(p-\frac{-3}{p}\right)\right) / 3} \equiv \begin{cases}2\left(\frac{-3}{p}\right)(\bmod p) & \text { if } y \equiv 0(\bmod 3), \\ -\left(\frac{-3}{p}\right)(\bmod p) & \text { if } y \neq \equiv(\bmod 3) .\end{cases}
$$

Remark 6.2. For the values of $P_{(p-1) / 2}(\bmod p)$ and $P_{(p+1) / 2}(\bmod p)$ one may consult [S1].

Corollary 6.6. Let $p$ be a prime greater than 3 . Then

(i) $p \mid P_{\left(p-\left(\frac{-3}{p}\right)\right) / 3}$ if and only if $p=x^{2}+54 y^{2}$ or $p=2 x^{2}+27 y^{2}$ for some integers $x$ and $y$ according as $p \equiv 1(\bmod 3)$ or $p \equiv 2(\bmod 3)$.

(ii) $p \mid P_{\left(p-\left(\frac{-3}{p}\right)\right) / 6}$ if and only if $p=x^{2}+216 y^{2}$ or $p=8 x^{2}+8 x y+29 y^{2}$ for some integers $x$ and $y$ according as $p \equiv 1(\bmod 3)$ or $p \equiv 2(\bmod 3)$.

The proof of Corollary 6.6 is similar to the proof of Corollary 6.5.

Remark 6.3. Let $p \equiv 1(\bmod 4)$ be a prime. From [L4], [S1] we know that $p \mid P_{(p-1) / 4}$ if and only if $p=x^{2}+32 y^{2}$ for some integers $x$ and $y$.

Finally, we discuss the Lucas sequence $\left\{u_{n}(1,4)\right\}$.

TheOREM 6.4. Let $p \equiv 1(\bmod 4)$ be a prime and hence $p=x^{2}+y^{2}$ for some integers $x$ and $y$. Then 


$$
\begin{aligned}
& u_{\left(p-\left(\frac{-3}{p}\right)\right) / 6}(1,4) \\
& \quad=2^{-\left(p-\left(\frac{-3}{p}\right)\right) / 6} u_{\left(p-\left(\frac{-3}{p}\right)\right) / 3}(-2,2) \\
& \quad \equiv \begin{cases}0(\bmod p) & \text { if } 9 \mid x y\left(x^{2}-y^{2}\right), \\
-\frac{1}{2}\left(\frac{2}{p}\right) \frac{x}{y}(\bmod p) & \text { if } x \equiv 2 y,-3 y, 4 y(\bmod 9) \text { or } y \equiv 3 x(\bmod 9)\end{cases}
\end{aligned}
$$

and

$$
\begin{aligned}
& v_{\left(p-\left(\frac{-3}{p}\right)\right) / 6}(1,4)=2^{-\left(p-\left(\frac{-3}{p}\right)\right) / 6} v_{\left(p-\left(\frac{-3}{p}\right)\right) / 3}(-2,2) \\
& \equiv \begin{cases}2\left(\frac{-6}{p}\right)(\bmod p) & \text { if } 9 \mid x y\left(x^{2}-y^{2}\right), \\
-\left(\frac{-6}{p}\right)(\bmod p) & \text { if } 9 \nmid x y\left(x^{2}-y^{2}\right) .\end{cases}
\end{aligned}
$$

Proof. By (6.3) and (6.4) we have

$$
\begin{aligned}
2^{n} u_{n}(1,4) & =\frac{1}{2 \sqrt{3}}\left((4+2 \sqrt{3})^{n}-(4-2 \sqrt{3})^{n}\right) \\
& =\frac{1}{2 \sqrt{3}}\left((1+\sqrt{3})^{2 n}-(1-\sqrt{3})^{2 n}\right)=u_{2 n}(-2,2)
\end{aligned}
$$

and

$$
\begin{aligned}
2^{n} v_{n}(1,4) & =(4+2 \sqrt{3})^{n}+(4-2 \sqrt{3})^{n} \\
& =(1+\sqrt{3})^{2 n}+(1-\sqrt{3})^{2 n}=v_{2 n}(-2,2)
\end{aligned}
$$

Since $\frac{3 x}{y} \cdot \frac{x}{y} \equiv-3(\bmod p)$ it follows from Proposition 2.2(i) that $x / y \in C_{i}(p)$ if and only if $3 x / y \in C_{i}(p)$. Thus, from $(6 x / y)^{2} \equiv-3\left(2^{2}-4(-2)\right)(\bmod p)$ and Theorem 6.1 we get

$$
u_{\left(p-\left(\frac{-3}{p}\right)\right) / 3}(-2,2) \equiv \begin{cases}0(\bmod p) & \text { if } x / y \in C_{0}(p), \\ \pm \frac{y}{2 x} \cdot 2^{-[p / 3]}(\bmod p) & \text { if } \pm x / y \in C_{1}(p)\end{cases}
$$

and

$$
v_{\left(p-\left(\frac{-3}{p}\right)\right) / 3}(-2,2) \equiv \begin{cases}2 \cdot(-2)^{-[p / 3]}(\bmod p) & \text { if } x / y \in C_{0}(p), \\ -(-2)^{-[p / 3]}(\bmod p) & \text { if } x / y \notin C_{0}(p) .\end{cases}
$$

From the proof of Theorem 5.2 we see that

$$
x / y \in C_{0}(p) \Leftrightarrow 9 \mid x y\left(x^{2}-y^{2}\right)
$$

and that

$$
x / y \in C_{1}(p) \Leftrightarrow x \equiv 2 y,-3 y, 4 y(\bmod 9) \text { or } y \equiv 3 x(\bmod 9) .
$$


Now, combining the above with the facts that

$(-1)^{[p / 3]}=\left(\frac{-3}{p}\right) \quad$ and $\quad 2^{\left.-\left(p-\frac{-3}{p}\right)\right) / 6} \cdot 2^{-[p / 3]}=2^{-(p-1) / 2} \equiv\left(\frac{2}{p}\right)(\bmod p)$ yields the desired result.

Corollary 6.7. Let $p>3$ be a prime. Then $p \mid u_{\left(p-\left(\frac{-3}{p}\right)\right) / 6}(1,4)$ (or $\left.p \mid u_{\left(p-\left(\frac{-3}{p}\right)\right) / 3}(-2,2)\right)$ if and only if $p$ can be represented by $x^{2}+81 y^{2}$ or $2 x^{2}+2 x y+41 y^{2}$ according as $p \equiv 1(\bmod 3)$ or $p \equiv 2(\bmod 3)$.

REMARK 6.4. Let $p>3$ be a prime. Using the method in the proof of Corollary 6.5(ii) one can similarly prove that $p \mid u_{\left.\left(p-\frac{(-3}{p}\right)\right) / 6}(-2,2)$ if and only if $p$ can be represented by $16 x^{2}+81 y^{2}, x^{2}+1296 y^{2}, 8 x^{2}+8 x y+41 y^{2}$ or $32 x^{2}-8 x y+41 y^{2}$.

Acknowledgements. I am grateful to the referee for suggesting many additional references.

\section{References}

[Ba] Ph. Barkan, Partitions quadratiques et cyclotomie, Sém. Delange-Pisot-Poitou (1975), Zbl324:10037.

[Bu] K. Burde, Zur Herleitung von Reziprozitätsgesetzen unter Benutzung von endlichen Körpern, J. Reine Angew. Math. 293/294 (1977), 418-427, MR57:16178.

[Ca] C. Cailler, Sur les congruences du troisième degré, Enseign. Math. 10 (1908), $474-487$.

[C] A. Cauchy, Sur la résolution des équivalences dont les modules se réduisent à des nombres premiers, Exercises de Mathématiques 4 (1829), 253-292.

[CG] A. Cunningham and T. Gosset, 4-tic and 3-bic residuacity-tables, Messenger Math. 50 (1920), 1-30.

[D] L. E. Dickson, History of the Theory of Numbers, Vol. I, Chelsea, New York, 1952, 393-407.

[FR] E. T. Federighi and R. G. Roll, Fibonacci Quart. (1966), 85-88.

[HW] R. H. Hudson and K. S. Williams, Resolution of ambiguities in the evaluation of cubic and quartic Jacobsthal sums, Pacific J. Math. 99 (1982), 379-386.

[IR] K. Ireland and M. Rosen, A Classical Introduction to Modern Number Theory, Springer, New York, 1982.

[J] J. G. D. Jacobi, De residuis cubicis commentatio numerosa, J. Reine Angew. Math. 2 (1827), 66-69.

[K] L. Kronecker, Werke, Vol. II, 93 and 97-101; Vol. IV, 123-129, Chelsea, New York, 1968.

[L1] E. Lehmer, Criteria for cubic and quartic residuacity, Mathematika 5 (1958), 20-29.

[L2] —, On Euler's criterion, J. Austral. Math. Soc. 1 (1959/1961, part 1), 64-70, MR21:7191.

[L3] - On the cubic character of quadratic units, J. Number Theory 5 (1973), 385389, MR48:271. 
[L4] E. Lehmer, On the quartic character of quadratic units, J. Reine Angew. Math. 268/269 (1974), 294-301.

[L5] - On the number of solutions of $u^{k}+D \equiv w(\bmod p)$, Pacific J. Math. 5 (1955), 103-118.

[L6] - On the quadratic character of the Fibonacci root, Fibonacci Quart. 4 (1966), 135-138, MR39:160.

[Li] H. von Lienen, Reelle kubische und biquadratische Legendre-Symbole, J. Reine Angew. Math. 305 (1979), 140-154.

[R] P. Ribenboim, The Book of Prime Number Records, 2nd ed., Springer, Berlin, 1989, 44-50.

[Sh] D. Shanks, On Gauss and composition I, in: Number Theory and Applications (edited by R. A. Mollin), Dordrecht, Boston, 1989, 163-178, MR92e:11150.

[SW] B. K. Spearman and K. S. Williams, The cubic congruence $x^{3}+A x^{2}+B x+C \equiv$ $0(\bmod p)$ and binary quadratic forms, J. London Math. Soc. (2) 46 (1992), 397410, MR93j:11004.

[St] L. Stickelberger, Über eine neue Eigenschaft der Diskriminanten algebraischer Zahlkörper, Verhand. I. Internat. Math. Kongress (1897), Zürich, 182-193.

[S1] Z. H. Sun, Combinatorial sum $\sum_{\substack{k=0 \\ k \equiv r(\bmod m)}}^{n}\left(\begin{array}{l}n \\ k\end{array}\right)$ and its applications in number theory II, J. Nanjing Univ. Biquarterly 10 (1993), 105-118, MR94j:11021.

[S2] - Supplements to the theory of biquadratic residues, submitted.

[SS] Z. H. Sun and Z. W. Sun, Fibonacci numbers and Fermat's last theorem, Acta Arith. 60 (1992), 371-388.

[We] P. J. Weinberger, The cubic character of quadratic units, Proc. 1972 Number Theory Conference, Univ. of Colorado, 1972, 241-242, MR52:10673.

[W1] K. S. Williams, On Euler's criterion for cubic non-residues, Proc. Amer. Math. Soc. 49 (1975), 277-283.

[W2] -, Cubic nonresidues $(\bmod p)$, Delta 6 (1976), 23-28, MR54:5095.

[WH] K. S. Williams and R. H. Hudson, Representation of primes by the principal form of discriminant $-D$ when the class number $h(-D)$ is 3, Acta Arith. 57 (1991), 131-153.

Department of Mathematics

Huaiyin Teachers College

Huaiyin 223001, Jiangsu, People's Republic of China

E-mail: zwsun@netra.nju.edu.cn

Received on 21.11.1994

and in revised form on 8.9.1997

$(2700)$ 